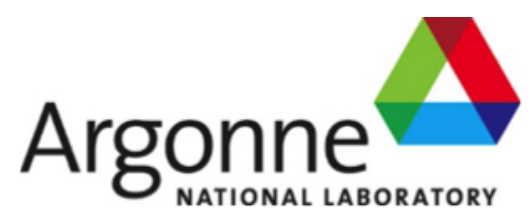

ANL-ART-94

\title{
FY17 Status Report on the Initial EPP Finite Element Analysis of Grade 91 Steel
}

Nuclear Engineering Division 
About Argonne National Laboratory

Argonne is a U.S. Department of Energy laboratory managed by UChicago Argonne, LLC under contract DE-AC02-06CH11357. The Laboratory's main facility is outside Chicago, at 9700 South Cass Avenue, Argonne, Illinois 60439. For information about Argonne and its pioneering science and technology programs, see www.anl.gov.

\section{DOCUMENT AVAILABILITY}

Online Access: U.S. Department of Energy (DOE) reports produced after 1991 and a growing number of pre-1991 documents are available free via DOE's SciTech Connect (http://www.osti.gov/scitech/)

Reports not in digital format may be purchased by the public from the National Technical Information Service (NTIS):

U.S. Department of Commerce

National Technical Information Service

5301 Shawnee Rd

Alexandria, VA 22312

www.ntis.gov

Phone: (800) 553-NTIS (6847) or (703)

605-6000 Fax: (703) 605-6900

Email: orders@ntis.gov

Reports not in digital format are available to DOE and DOE contractors from the Office of Scientific and Technical Information (OSTI):

U.S. Department of Energy

Office of Scientific and Technical Information

P.O. Box 62

Oak Ridge, TN 37831-0062

www.osti.gov

Phone: (865) 576-8401

Fax: (865) 576-5728

Email: reports@osti.gov

\section{Disclaimer}

This report was prepared as an account of work sponsored by an agency of the United States Government. Neither the United States Government nor any agency thereof, nor UChicago Argonne, LLC, nor any of their employees or officers, makes any warranty, express or implied, or assumes any legal liability or responsibility for the accuracy, completeness, or usefulness of any information, apparatus, product, or process disclosed, or represents that its use would not infringe privately owned rights. Reference herein to any specific commercial product, process, or service by trade name, trademark, manufacturer, or otherwise, does not necessarily constitute or imply its endorsement, recommendation, or favoring by the United States Government or any agency thereof. The views and opinions of document authors expressed herein do not necessarily state or reflect those of the United States Government or any agency thereof, Argonne National Laboratory, or UChicago Argonne, LLC. 


\section{FY17 Status Report on the Initial EPP Finite Element Analysis of Grade 91 Steel}

Nuclear Engineering Division

Argonne National Laboratory

July 2017

Prepared by

M. C. Messner, Argonne National Laboratory

T.-L. Sham, Argonne National Laboratory 



\section{Abstract}

This report describes a modification to the elastic-perfectly plastic (EPP) strain limits design method to account for cyclic softening in Gr. 91 steel. The report demonstrates that the unmodified EPP strain limits method described in current ASME code case is not conservative for materials with substantial cyclic softening behavior like Gr. 91 steel. However, the EPP strain limits method can be modified to be conservative for softening materials by using softened isochronous stress-strain curves in place of the standard curves developed from unsoftened creep experiments. The report provides softened curves derived from inelastic material simulations and factors describing the transformation of unsoftened curves to a softened state. Furthermore, the report outlines a method for deriving these factors directly from creep/fatigue tests. If the material softening saturates the proposed EPP strain limits method can be further simplified, providing a methodology based on temperature-dependent softening factors that could be implemented in an ASME code case allowing the use of the EPP strain limits method with Gr. 91. Finally, the report demonstrates the conservatism of the modified method when applied to inelastic simulation results and two bar experiments. 



\section{Table of Contents}

Abstract i i

Table of Contents $\quad$ iii

List of Figures $\quad$ V

List of Tables vii

1 Introduction 1

1.1 Background . . . . . . . . . . . . . . . . . . . . 1

1.2 EPP strain limits and creep/fatigue damage methods . . . . . . . . . . . . 1

2 Drucker's postulate and the EPP bounding theorem 5

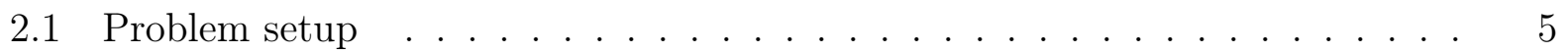

2.2 Steady state behavior . . . . . . . . . . . . . . . . . 8

2.3 Bounding the creep/plasticity solution with the cyclic plasticity solution _ . 11

2.4 Dissipation bounds to work bounds . . . . . . . . . . . . . . 13

2.5 Bounding the cyclic plasticity solution with an elastic-perfectly plastic solution based on the isochronous curve . . . . . . . . . . . . . . . . . . . 14

2.6 A summary of the EPP method with softening materials . . . . . . . . . 16

3 A modeling approach to establishing reduction factors 19

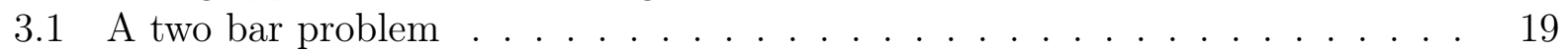

3.2 Simulating softened isochronous curves . . . . . . . . . . . . . . . . . . . 22

3.3 Calculating softening factors . . . . . . . . . . . . . . . . 23

3.4 A modified EPP strain limits procedure . . . . . . . . . . . . . . . . 25

3.4.1 Procedure requiring strain range and cycle count . . . . . . . . . 25

3.4.2 Simplified procedure requiring saturating softening behavior . . . . . 26

4 Experimental reduction factors 29

4.1 Proposed experiment type . . . . . . . . . . . . . . . . . 29

4.2 Calculating approximate softening factors from experimental data . . . . . 30

4.3 Saturating softening . . . . . . . . . . . . . . . 31

5 Grade 91 two bar tests 35

5.1 Test $\operatorname{setup} \ldots \ldots \ldots \ldots \ldots \ldots$

5.2 Comparison to modified EPP strain limits procedure . . . . . . . . . . . 35

6 Conclusions and future work 39

Acknowledgments $\quad 41$

Bibliography

$\begin{array}{ll}\text { Distribution list } & 47\end{array}$

ANL-ART-94 iii 



\section{List of Figures}

1.1 Plots illustrating G. 91 cyclic softening behavior at low and high temperatures.

2.1 Example model showing that non-Drucker stable models can capture Gr. 91 cyclic softening behavior. . . . . . . . . . . . . . . .

2.2 A schematic showing how 1) for a simple case the creep dissipation and the work done by an equivalent, rigid plastic model with flow stress given by the isochronous curve is the same and 2) the work of the equivalent plastic case can be bounded by a perfectly plastic material with a yield stress selected to match the value of the isochronous curve at the final strain value. . . . . .

3.1 Example cyclic softening simulation using the inelastic model. This curve shows the maximum stress versus cycle count for fully-reversed strain controlled loading with $\Delta \varepsilon=0.01$ at $T=600^{\circ} \mathrm{C}$ - the maximum cycle temperature. The model captures cyclic softening and predicts a saturating softening response - eventually the processes causing softening saturate and the material achieves a constant flow stress. . . . . . . . . . . . . . . . . . 20

3.2 Example two bar geometry. . . . . . . . . . . . . . . . . . . 20

3.3 Two bar temperature cycles. The top, no-hold cycle is a special case of the generic cycle on the bottom of the figure. . . . . . . . . . . . . . .

3.4 Plot illustrating the region the conservatism and non-conservatism of the unmodified EPP procedure versus inelastic simulation results. The dashed line indicates the tradeoff condition - below this line, in the region of high cyclic softening - the unmodified strain limits procedure is not conservative. . . .

3.5 Softened isochronous curves computed for the Gr. 91 inelastic model using a cycle strain range of $\Delta \varepsilon=1 \%$ at a temperature of $600^{\circ} \mathrm{C}$. The subfigure captions give the number of cycles. From the top of each plot to the bottom the lines represent isochrons of 1 hour, 10 hours, 100 hours, 1000 hours, 10000 hours, and 100000 hours. . . . . . . . . . . . . . . . 24

3.6 Plots illustrating general trends in the softening reduction factors. . . . . . 26

4.1 Data collected from fatigue/creep tests. The softened curve is from ORNL experimental work while the reference curve is from [18]. . . . . . . . . . . .

4.2 Plot showing the scaled isochronous curves, using the procedure described in this section, the ORNL experimental data, and the ASME isochronous reference curves. The figure shows the softened curves for 200, 400, 600, 800, and 1000 cycles, in addition to the reference curve. The arrow on the diagram shows how a softening factor can be computed from this data, using the $1 \%$ strain method developed above. The kinks in the softened curves is interpolation error. The ASME code only provides curves for a finite set of design lives, requiring interpolation to lives in between these values. . . . . . 32

4.3 Plots illustrating G. 91 cyclic softening behavior at low and high temperature. 33

5.1 Two bar experimental thermal profile. . . . . . . . . . . . . 36 



\section{List of Tables}

3.1 Two bar problem example loading parameters. . . . . . . . . . . . . . . 21

3.2 Design lives computed via inelastic analysis, for different values of $t_{\text {hold }}$. . . 21

3.3 Saturated softening factors computed from the inelastic numerical experiments. . . . . . . . . . . . . . . . . . . . . 27

4.1 Table comparing the experimental softening factors to the model-based softening factors for $600^{\circ} \mathrm{C}, 100,1000$, and 10000 hours life, and various numbers of cycles. The experimental factors were computed from ORNL data for the softened case and reference creep data sourced from [18]. . . . . . . . . . . .

4.2 Table comparing the experimental softening factors to the model-based softening factors for $550^{\circ} \mathrm{C}, 100,1000$, and 10000 hours life, and various numbers of cycles. The experimental factors were computed from ORNL data for the softened case and reference creep data sourced from [18] . . . . . . . . .

5.1 Comparison of experimental two bar test results to unmodified and modified EPP strain limits calculations. 



\section{Introduction}

\section{$1.1 \quad$ Background}

ASME Section III Division 5, Subsection HB, Subpart B provides construction rules for Class A metallic components under elevated temperature service. There are currently five Division 5 Class A materials qualified for 300,000-hour design life: $304 \mathrm{H}$ and $316 \mathrm{H}$ stainless steels, Alloy $800 \mathrm{H}$ austenitic steel, $2 \frac{1}{4} \mathrm{Cr}-1 \mathrm{Mo}$ ferritic steel, and $9 \mathrm{Cr}-1 \mathrm{Mo}-\mathrm{V}$ (Grade 91) ferriticmartensitic steel. Extension of allowable stresses and design parameters for Grade 91 steel to support 60-year (nominally 500,000 hours) design lives is one of the high priority R\&D items of the ASME Code committee responsible for Division 5.

The tasks required to extend the Grade 91 (Gr. 91) code rules for 500,000-hour design life include:

- extend time dependent allowable stresses to 500,000 hours;

- extend stress-to-rupture values to 500,000 hours;

- extend stress rupture factors to 500,000 hours;

- extend isochronous stress-strain curves to 500,000 hours;

- extend time-temperature limits for application of Section II external pressure charts to 500,000 hours;

- viscoplastic material model development;

- qualify the elastic perfectly-plastic (EPP) methods for strain limits and creep-fatigue damage evaluation of Gr. 91 components.

The focus of this work is the qualification of the EPP methods for Gr. 91.

\subsection{EPP strain limits and creep/fatigue damage methods}

Simplified design rules based on using elastic analysis results to satisfy deformation limits for Class A components are provided in Division 5 Appendix HBB-T. These bounding methods were established as conservative screening tools and the expectation was that inelastic analyses would sometimes be required. The technical basis of the simplified design methods was developed with the tacit assumption that plastic and creep deformation are uncoupled. When such conditions are not met, e.g., at higher temperatures, the technical basis of the simplified design rules is no longer valid.

The elastic, perfectly plastic methods were developed as bounding methods for strain limits and creep-fatigue evaluations to address this deficiency. The EPP methods are valid for the full range of allowable temperatures, even when plasticity and creep are coupled. Furthermore, they are tailored to modern finite element analysis tools, with no stress classification, no restrictions on geometry or loading, and account for redundant load paths.

As the name suggests the EPP methods require only an elastic-perfectly plastic analysis of a component using a pseudo-yield stress defined in the code case procedures. The methods are setup as screening checks, with the goal of allowing designers to quickly evaluate 


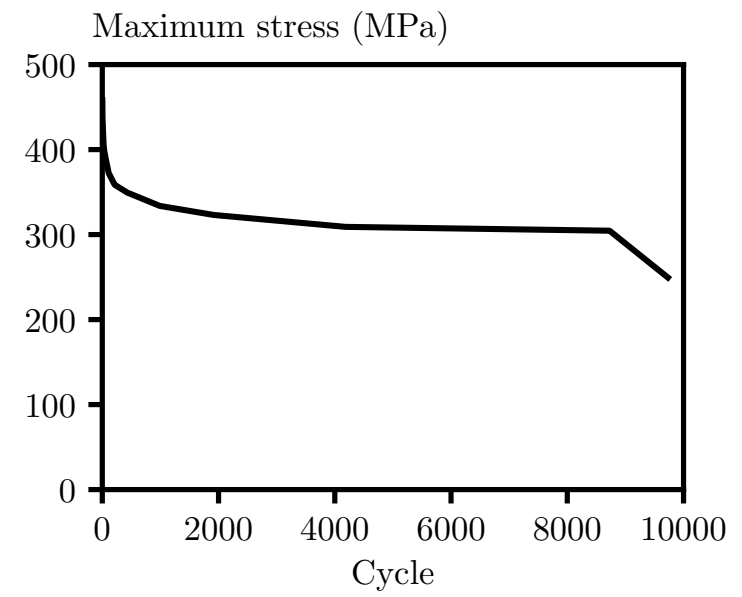

(a) $\mathrm{T}=320^{\circ} \mathrm{C}$ and $\Delta \varepsilon=0.7 \%[17]$.

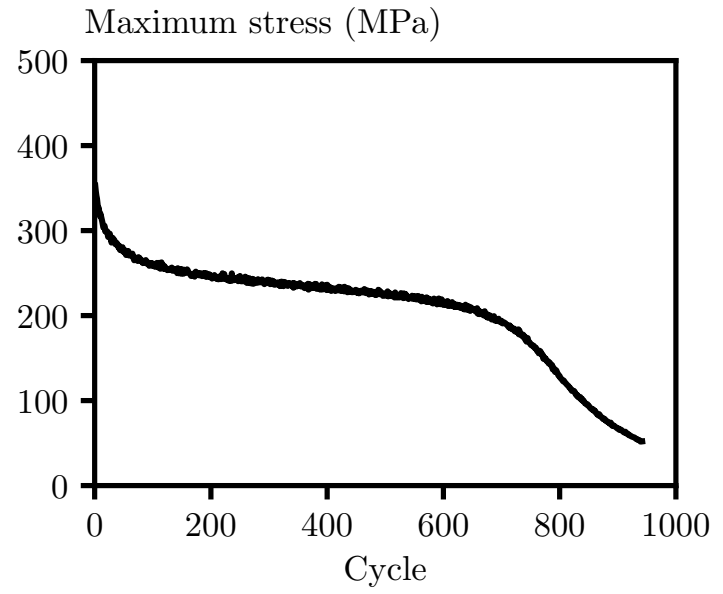

(b) $T=600^{\circ} \mathrm{C}$ and $\Delta \varepsilon=1 \%$ (personal communication with M. Li, Argonne National Laboratory).

Figure 1.1: Plots illustrating G. 91 cyclic softening behavior at low and high temperatures.

competing designs. Two ASME code cases, one each for strain limits [5] and creep fatigue damage [6] describe the EPP methodology for $304 \mathrm{H}$ and $316 \mathrm{H}$ stainless steel. Numerous reports published in the technical literature attest to the method's conservatism for stainless steel materials $[13,14,12,28,29]$.

The underpinnings of the EPP method are a series of bounds on the creep dissipation and external work of creeping structures first begun in the early 1970s by Ponter [21, 22, 23], extended by Ainsworth [2, 3, 4], and synthesized and extended further by Carter [9, 10, 11]. As shown in Chapter 2 of this report, tacitly these bounding theorems assume a cyclic hardening material response. Some ASME Division 5 Class A materials, like $304 \mathrm{H}$ and $316 \mathrm{H}$, meet this criterion. However, other Division 5 materials do not show cyclic hardening at elevated temperatures.

Gr. 91 is a modified 9Cr-1Mo ferritic/martensitic steel developed by Combustion Engineering and Oak Ridge National Laboratory for high temperature applications [25, 27]. The alloy has good creep strength at elevated temperatures [26] making it a prime candidate material for intermediate heat exchanger (IHX) use in sodium fast reactors designs.

Gr. 91 softens under both cyclic and monotonic load, particularly at high temperatures. Figure 1.1 shows experimental fatigue data on Gr. 91 from two sources illustrating the response of the material under repeated strain cycling [17]. At both intermediate and high temperatures Gr. 91 softens significantly during the test. This softening is caused by microstructural relaxation, particularly the rearrangement of the dislocation structure and a corresponding reduction in dislocation density but also migration of carbides and growth of sub-grains [1, 15, 24]. This reports addresses the applicability of the EPP strain limits method to softening materials and to Gr. 91 steel in particular. Future work will address creep-fatigue.

Chapter 2 demonstrates the failure of the formal derivation of the EPP bounding theorems for cyclic softening materials. The next chapter illustrates the non-conservatism of 
the EPP strain limits code case for Gr. 91 structures experiencing high numbers of load cycles over their service life and proposes a modification to the methodology using softened isochronous curves, described by a softening factor, to select the EPP pseuedo-yield stress. This chapter demonstrates that with this modification the EPP strain limits method again becomes conservative. These results are based on inelastic simulation results; Chapter 4 shows how the softening factors can instead be derived from creep-fatigue experiments. Furthermore, this chapter speculates on the saturation of cyclic softening in Gr. 91 and shows that, provided the material softening behavior actually saturates, the modification to the EPP strain limits method proposed here can be further simplified. This final method requires only the definition and application of a temperature-dependent softening factor, rather than a softening factor that depends on temperature, strain range, and cycle count. The method with this simplification could serve as the basis for an ASME code case allowing the use of the EPP strain limits method on Gr. 91 components. Chapter 5 demonstrates the conservatism of the modified EPP method against a series of two bar experiments. The final chapter summarizes the report and outlines future work, e.g. applying this concept to the EPP creep-fatigue damage evaluation method. 



\section{Drucker's postulate and the EPP bounding theorem}

The EPP strain limits method relies on a chain of reasoning to bound a steady state solution to problem involving creep and plasticity (the SS solution) by a cyclic plasticity solution using an elastic-plastic material with a pseudo-yield stress selected by referencing the material's isochronous curve at an appropriate design life (the EPP solution). In order to assess the impact of cyclic softening on the EPP methodology this section re-derives the bounds leading to the EPP method. The derivation highlights potential failures of the bounding theorem that could develop in softening materials. This section derives only the simplest, most restrictive form of the bounding theorems, sufficient to illustrate the problems that softening may cause, and cites the literature for extensions to more complex material constitutive response.

In the original work by Carter $[10,11]$ he does not attempt to the full bounding theorem rigorously, instead relying on an intuitive appeal to the idea that decreasing the yield stress in a plastic analysis does not decrease the deformation. This work attempts to prove the bound required for the second step rigorously, albeit for a highly simplified case in order to better establish the theoretical framework of the EPP strain limits design method and to determine what effect softening might have on this stage of the derivation.

\subsection{Problem setup}

Consider a body $\Omega$ with $\boldsymbol{\sigma} \cdot \boldsymbol{n}=\hat{\boldsymbol{t}}(t)$ on $\partial \Omega_{n}$ and $\boldsymbol{u}=\mathbf{0}$ on $\partial \Omega_{e}$ such that $\partial \Omega_{n} \cap \partial \Omega_{e}=\emptyset$, $\partial \Omega_{n} \cup \partial \Omega_{e}=\partial \Omega$, and the tractions $\hat{\boldsymbol{t}}(t)$ and the temperature field $\theta(t)$ are periodic with period $\tau$. Divide the strain rate into

$$
\dot{\varepsilon}=\dot{\varepsilon}_{e}+\dot{\varepsilon}_{p}+\dot{\varepsilon}_{c}+\dot{\varepsilon}_{t}
$$

with

$$
\begin{aligned}
& \boldsymbol{\varepsilon}_{e}=\boldsymbol{S}: \boldsymbol{\sigma} \\
& \dot{\varepsilon}_{p}=\dot{\gamma} \frac{\partial f}{\partial \boldsymbol{\sigma}} \\
& \dot{\boldsymbol{\varepsilon}}_{c}=\phi^{n} \frac{\partial \phi}{\partial \boldsymbol{\sigma}}
\end{aligned}
$$

where $\phi$ is convex, homogeneous, and degree one in $\boldsymbol{\sigma}$, and $n>0$ and

$$
\varepsilon_{t}=\alpha\left(\theta-\theta_{0}\right) \boldsymbol{I}
$$

Note the creep dissipation is:

$$
\dot{D}=\boldsymbol{\sigma}: \dot{\boldsymbol{\varepsilon}}_{c}=\boldsymbol{\sigma}: \phi^{n} \frac{\partial \phi}{\partial \boldsymbol{\sigma}}=\boldsymbol{\sigma}: \frac{1}{n+1} \frac{\partial}{\partial \boldsymbol{\sigma}}\left(\phi^{n+1}\right)=\phi^{n+1}(\boldsymbol{\sigma}) .
$$

Assume the plasticity law obeys Drucker's postulate such that

$$
\left(\boldsymbol{\sigma}-\boldsymbol{\sigma}^{\star}\right): \dot{\varepsilon}_{p} \geq 0
$$


for any plastically admissible stress state $\boldsymbol{\sigma}^{\star}$. The starred and unstarred quantities can be interchanged and so Drucker's postulate (Eq. 2.7) recast as:

$$
\begin{gathered}
\left(\boldsymbol{\sigma}-\boldsymbol{\sigma}^{\star}\right): \dot{\varepsilon}_{p}+\left(\boldsymbol{\sigma}^{\star}-\boldsymbol{\sigma}\right): \dot{\varepsilon}_{p}^{\star} \geq 0 \\
\left(\boldsymbol{\sigma}-\boldsymbol{\sigma}^{\star}\right): \dot{\varepsilon}_{p}-\left(\boldsymbol{\sigma}-\boldsymbol{\sigma}^{\star}\right): \dot{\varepsilon}_{p}^{\star} \geq 0 \\
\left(\boldsymbol{\sigma}-\boldsymbol{\sigma}^{\star}\right):\left(\dot{\varepsilon}_{p}-\dot{\boldsymbol{\varepsilon}}_{p}^{\star}\right) \geq 0
\end{gathered}
$$

A similar expression can be developed for the creep law. By convexity

$$
\phi^{n+1}(\boldsymbol{\sigma}) \geq \phi^{n+1}\left(\boldsymbol{\sigma}^{\star}\right)+(n+1) \phi^{\star n} \frac{\partial \phi^{\star}}{\partial \boldsymbol{\sigma}}:\left(\boldsymbol{\sigma}-\boldsymbol{\sigma}^{\star}\right) .
$$

Subtract $\boldsymbol{\sigma}^{\star}: \dot{\varepsilon}_{c}^{\star}$ :

$$
\phi^{n+1}(\boldsymbol{\sigma})-\boldsymbol{\sigma}^{\star}: \dot{\varepsilon}_{c}^{\star} \geq \phi^{n+1}\left(\boldsymbol{\sigma}^{\star}\right)+(n+1) \phi^{\star n} \frac{\partial \phi^{\star}}{\partial \boldsymbol{\sigma}}:\left(\boldsymbol{\sigma}-\boldsymbol{\sigma}^{\star}\right)-\boldsymbol{\sigma}^{\star}: \dot{\varepsilon}_{c}^{\star} .
$$

Or

$$
\dot{D}(\boldsymbol{\sigma})-\dot{D}\left(\boldsymbol{\sigma}^{\star}\right) \geq \phi^{n+1}\left(\boldsymbol{\sigma}^{\star}\right)+(n+1) \dot{\varepsilon}_{c}^{\star}:\left(\boldsymbol{\sigma}-\boldsymbol{\sigma}^{\star}\right)-\phi^{n+1}\left(\boldsymbol{\sigma}^{\star}\right) .
$$

And so

$$
\dot{D}(\boldsymbol{\sigma})-\dot{D}\left(\boldsymbol{\sigma}^{\star}\right) \geq(n+1)\left(\boldsymbol{\sigma}-\boldsymbol{\sigma}^{\star}\right): \dot{\varepsilon}_{c}^{\star}
$$

Again the starred and unstarred quantities can be interchanged and so

$$
\begin{gathered}
\dot{D}(\boldsymbol{\sigma})-\dot{D}\left(\boldsymbol{\sigma}^{\star}\right)+\dot{D}\left(\boldsymbol{\sigma}^{\star}\right)-\dot{D}(\boldsymbol{\sigma}) \geq(n+1)\left(\boldsymbol{\sigma}-\boldsymbol{\sigma}^{\star}\right): \dot{\varepsilon}_{c}^{\star}+(n+1)\left(\boldsymbol{\sigma}^{\star}-\boldsymbol{\sigma}\right): \dot{\varepsilon}_{c} \\
0 \geq(n+1)\left(\boldsymbol{\sigma}-\boldsymbol{\sigma}^{\star}\right): \dot{\varepsilon}_{c}^{\star}+(n+1)\left(\boldsymbol{\sigma}^{\star}-\boldsymbol{\sigma}\right): \dot{\varepsilon}_{c} \\
(n+1)\left(\boldsymbol{\sigma}-\boldsymbol{\sigma}^{\star}\right):\left(\dot{\varepsilon}_{c}-\dot{\varepsilon}_{c}^{\star}\right) \geq 0 \\
\left(\boldsymbol{\sigma}-\boldsymbol{\sigma}^{\star}\right):\left(\dot{\varepsilon}_{c}-\dot{\varepsilon}_{c}^{\star}\right) \geq 0 .
\end{gathered}
$$

In Eq. 2.10 the $*$ states must be plastically admissible but Eq. 2.18 has no such restriction.

The above describes the most general form of model considered here. Below this full model will be referred to as the creep/plasticity model and the corresponding solution under periodic loading referred to as the creep/plasticity solution. Throughout the course of the derivation several simplified structural models will also be considered. The first is a model with $\dot{\varepsilon}_{c}=\mathbf{0}$ but retaining the remainder of the above definition. This system will be called the cyclic plasticity model. A subsequent representation will consider a rigid plastic solution $\left(\dot{\boldsymbol{\varepsilon}}_{e}=\mathbf{0}\right)$ and alter the definition of the plasticity model so that the uniaxial flow stress matches an isochronous stress-strain curve. This will be the rigid plastic model. Finally, the derivation will consider a perfectly-plastic solution with a yield stress selected from an isochronous stress-strain curve. This will be labeled the EPP model.

Grade 91 softens under monotonic loading at elevated temperatures and under straincontrolled cyclic loading at moderate and elevated temperatures. In general, softening materials may not obey Drucker's postulate. To be more precise, consider the 1D plastic material 
with associated flow and hardening described by

$$
\begin{array}{rlr}
\dot{\varepsilon} & = & \frac{\dot{\sigma}}{E}+\dot{\varepsilon}_{p} \\
\dot{\varepsilon}_{p} & = & \dot{\gamma} \frac{\partial f(\sigma, \boldsymbol{h})}{\partial \sigma} \\
f(\sigma, \boldsymbol{h}) & = & 0 \\
\dot{\boldsymbol{h}} & = & \dot{\gamma} \boldsymbol{H}(\sigma, \boldsymbol{h})
\end{array}
$$

and the usual consistency conditions for plastic flow. Here $\boldsymbol{h}$ is some generic set of internal variables. Then

$$
\dot{f}=\frac{\partial f}{\partial \sigma} \dot{\sigma}+\frac{\partial f}{\partial \boldsymbol{h}} \cdot \dot{\boldsymbol{h}}=f_{\sigma} \dot{\sigma}+f_{\boldsymbol{h}} \cdot \dot{\boldsymbol{h}}=0
$$

and

$$
\dot{\gamma}=\frac{E f_{\sigma} \dot{\varepsilon}}{E f_{\sigma} f_{\sigma}-\boldsymbol{f}_{h} \cdot \boldsymbol{H}} .
$$

Drucker's inequality can then be stated as

$$
\begin{array}{r}
\dot{\sigma} \dot{\varepsilon}_{p}>0 \\
-\boldsymbol{f}_{h} \cdot \boldsymbol{H}>0 .
\end{array}
$$

Consider two specific cases, the first where

$$
\begin{aligned}
& \boldsymbol{h}=\quad[\alpha] \\
& \boldsymbol{H}=[1] \\
& f=\quad|\sigma|-H \alpha-k
\end{aligned}
$$

then

$$
M_{p}=-\boldsymbol{f}_{h} \cdot \boldsymbol{H}=H
$$

and Drucker's postulate holds for

$$
H>0
$$

i.e. isotropic hardening. For the second case consider linear isotropic and kinematic motion of the yield surface by letting

$$
\begin{array}{rrr}
\boldsymbol{h} & = & {\left[\begin{array}{ll}
\alpha & \beta
\end{array}\right]} \\
\boldsymbol{H} & = & {\left[\begin{array}{rr}
1 & \operatorname{sign}(\sigma-J \beta)
\end{array}\right]} \\
f & = & |\sigma-J \beta|-H \alpha-k
\end{array}
$$

then

$$
M_{p}=-\boldsymbol{f}_{h} \cdot \boldsymbol{H}=H+J
$$

and Drucker's postulate holds for

$$
H+J>0 .
$$

So the sum of the isotropic and kinematic modulii must be greater than zero - but this does not preclude one of the modulii from being less than zero. In general, the term $-\boldsymbol{f}_{h} \cdot \boldsymbol{H}$ 


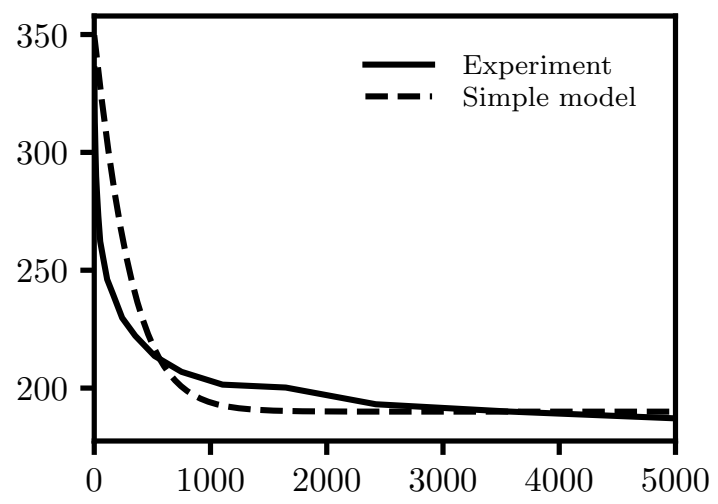

Figure 2.1: Example model showing that non-Drucker stable models can capture Gr. 91 cyclic softening behavior.

could be called the generalized plastic modulus and Drucker's postulate holds only if it is positive for all material states.

Figure 2.1 shows that aspects of the behavior of Gr. 91 can be fit with models that have a negative generalized plastic modulus. This figure shows a cyclic softening curve from [17] overlaid with a simple, rate independent plasticity model capturing the gross features of the experimental data. This model has Voce isotropic softening of the form:

$$
\begin{array}{rrr}
\boldsymbol{h} & = & {[\alpha]} \\
\boldsymbol{H} & = & {[1]} \\
f & = & |\sigma|-R\left(1-e^{-b \alpha}\right)-k \\
M_{p} & = & b R e^{-b \alpha}
\end{array}
$$

which is Drucker stable if $b R>0$. The Voce model used in the figure to describe softening has $R=-160 \mathrm{MPa}$ and $b=0.02$ and so the model is not Drucker stable. This does not mean that the response of Gr. 91 cannot be captured with a stable model or that the response of the material itself is unstable. However, it does demonstrate there is the possibility that the material's constitutive response does not obey the classical stability criteria.

Therefore, in Gr. 91 - which has both monotonic and cyclic softening - Eqs. 2.7 and 2.10 may not hold. Furthermore, the convex creep model used above may not describe the response of Gr. 91 and there is no guarantee that convexity, and hence Eqs. 2.10 and 2.18 will hold for the real material. Losing these inequalities will affect several of the theoretical results developed below and will be re-emphasized in the course of the derivation.

\subsection{Steady state behavior}

The bounds assume that under the imposed cyclic tractions and temperatures both the creep/plasticity response of the structure and the several plastic responses come to a steady cyclic solution. When the structure achieves a steady cyclic response the stresses and strain 
rate fields are periodic over the whole domain. However, the displacement and strain fields need not be periodic.

In the steady state regime structures fall into one of four categories:

1. elastic - the model never deforms plastically;

2. elastic shakedown - the structure undergoes initial plastic deformation but the steady state solution is purely elastic;

3. plastic shakedown - the steady state solution includes plasticity but the strain and displacements fields are periodic

4. ratcheting - the steady state solution strains and displacements are not periodic, the structure continues to accumulate deformation without bound.

The first three states are safe in that deformation in the structure will be bounded as it undergoes additional load cycles.

This section proves that for the problem described above the stress field, and consequentially the strain rate field, becomes periodic under cyclic tractions. This derivation is originally due to Frederick and Armstrong [16], with extensions to more complicated constitutive response by $[21,20,8]$. However, this theorem will not apply to materials that do not obey Drucker's postulate, the implications of which are discussed below.

Theorem. For $t \geq t^{\star} \boldsymbol{\sigma}(t+\tau)=\boldsymbol{\sigma}(t)$.

Proof. In this proof let $\boldsymbol{\sigma}=\boldsymbol{\sigma}(t), \boldsymbol{\sigma}^{\star}=\boldsymbol{\sigma}(t+\tau)$, and so on for other starred and unstarred quantities.

Consider a positive-definite measure of the difference in the stresses

$$
d=\frac{1}{2} \int_{\Omega}\left(\boldsymbol{\sigma}^{\star}-\boldsymbol{\sigma}\right): \boldsymbol{S}:\left(\boldsymbol{\sigma}^{\star}-\boldsymbol{\sigma}\right) d V \geq 0
$$

which is zero if the stresses are cyclic. The time rate of change is

$$
\begin{gathered}
\dot{d}=\int_{\Omega}\left(\boldsymbol{\sigma}^{\star}-\boldsymbol{\sigma}\right): \boldsymbol{S}:\left(\dot{\boldsymbol{\sigma}}^{\star}-\dot{\boldsymbol{\sigma}}\right) d V \\
\dot{d}=\int_{\Omega}\left(\boldsymbol{\sigma}^{\star}-\boldsymbol{\sigma}\right):\left(\dot{\varepsilon}_{e}^{\star}-\dot{\boldsymbol{\varepsilon}}_{e}\right) d V .
\end{gathered}
$$

Substituting the strains

$$
\dot{d}=\int_{\Omega}\left(\boldsymbol{\sigma}^{\star}-\boldsymbol{\sigma}\right):\left(\left\{\dot{\varepsilon}^{\star}-\dot{\varepsilon}_{p}^{\star}-\dot{\varepsilon}_{c}^{\star}-\dot{\varepsilon}_{t}^{\star}\right\}-\left\{\dot{\varepsilon}-\dot{\varepsilon}_{p}-\dot{\varepsilon}_{c}-\dot{\varepsilon}_{t}\right\}\right) d V .
$$

The thermal strain rates are periodic because the temperature field is periodic

$$
\dot{d}=\int_{\Omega}\left(\boldsymbol{\sigma}^{\star}-\boldsymbol{\sigma}\right):\left(\left\{\dot{\varepsilon}^{\star}-\dot{\varepsilon}_{p}^{\star}-\dot{\varepsilon}_{c}^{\star}\right\}-\left\{\dot{\varepsilon}-\dot{\varepsilon}_{p}-\dot{\varepsilon}_{c}\right\}\right) d V
$$


which we separate out into components

$$
\dot{d}=\int_{\Omega}\left(\boldsymbol{\sigma}^{\star}-\boldsymbol{\sigma}\right):\left(\dot{\varepsilon}^{\star}-\dot{\varepsilon}\right) d V-\int_{\Omega}\left(\boldsymbol{\sigma}^{\star}-\boldsymbol{\sigma}\right):\left(\dot{\varepsilon}_{p}^{\star}-\dot{\varepsilon}_{p}\right) d V-\int_{\Omega}\left(\boldsymbol{\sigma}^{\star}-\boldsymbol{\sigma}\right):\left(\dot{\varepsilon}_{c}^{\star}-\dot{\varepsilon}_{c}\right) d V
$$

Both stresses $\boldsymbol{\sigma}^{\star}$ and $\boldsymbol{\sigma}$ are in equilibrium with the same external loads because the tractions are periodic and both the total strain rate fields are compatible by definition so we can apply virtual power to eliminate the first term:

$$
\int_{\Omega}\left(\boldsymbol{\sigma}^{\star}-\boldsymbol{\sigma}\right):\left(\dot{\varepsilon}^{\star}-\dot{\varepsilon}\right) d V=\int_{\partial \Omega_{n}}\left(\boldsymbol{t} \cdot \dot{\boldsymbol{u}}^{\star}+\boldsymbol{t} \cdot \dot{\boldsymbol{u}}-\boldsymbol{t} \cdot \dot{\boldsymbol{u}}^{\star}-\boldsymbol{t} \cdot \dot{\boldsymbol{u}}\right) d V=0
$$

leaving

$$
\dot{d}=-\int_{\Omega}\left(\boldsymbol{\sigma}^{\star}-\boldsymbol{\sigma}\right):\left(\dot{\varepsilon}_{p}^{\star}-\dot{\varepsilon}_{p}\right) d V-\int_{\Omega}\left(\boldsymbol{\sigma}^{\star}-\boldsymbol{\sigma}\right):\left(\dot{\varepsilon}_{c}^{\star}-\dot{\varepsilon}_{c}\right) d V .
$$

By Eqs. 2.10 and 2.18 both $\int_{\Omega}\left(\boldsymbol{\sigma}^{\star}-\boldsymbol{\sigma}\right):\left(\dot{\varepsilon}_{p}^{\star}-\dot{\boldsymbol{\varepsilon}}_{p}\right) d V \geq 0$ and $\int_{\Omega}\left(\boldsymbol{\sigma}^{\star}-\boldsymbol{\sigma}\right):\left(\dot{\boldsymbol{\varepsilon}}_{c}^{\star}-\dot{\boldsymbol{\varepsilon}}_{c}\right) d V \geq$ 0 and so

$$
\dot{d} \leq 0
$$

But $d$ is positive definite and $\dot{d}<0$ which means $\dot{d} \rightarrow 0$ so that $\exists t^{\star}$ such that $\dot{d}(t)=0 \forall t \geq t^{\star}$

$$
\sigma=\sigma^{\star}
$$

A periodic stress field implies a periodic strain rate field and periodic plastic and creep strain rate fields. Note that the creep strain rate field (Eq. 2.4) is solely a function of the stress field. Therefore a periodic stress field implies a periodic creep strain rate field:

$$
\dot{\varepsilon}_{c}=\dot{\varepsilon}_{c}^{\star}
$$

Similarly, periodic stresses implies

$$
\dot{\varepsilon}_{p}=\dot{\varepsilon}_{p}^{\star}
$$

by the classical uniqueness theorem.

Periodic stresses

$$
\dot{\boldsymbol{\sigma}}=\dot{\boldsymbol{\sigma}}^{\star}
$$

implies the elastic strains

$$
\varepsilon_{e}=\varepsilon_{e}^{\star}
$$

and strain rates are periodic

$$
\dot{\varepsilon}_{e}=\dot{\varepsilon}_{e}^{\star}
$$

and we know by periodicity of the temperature field that the thermal strains and strain rates are periodic

$$
\begin{aligned}
\varepsilon_{t} & =\varepsilon_{t}^{\star} \\
\dot{\varepsilon}_{t} & =\dot{\varepsilon}_{t}^{\star}
\end{aligned}
$$


Now because each individual rate in the decomposition of the total strain rate field (Eq. 2.1 ) is periodic the total strain rate field is periodic

$$
\dot{\varepsilon}=\dot{\varepsilon}^{\star} \text {. }
$$

Drucker's stability postulate and the equivalent inequality developed through convexity of the creep law plays a key role in this proof and, as noted above, softening materials may not obey Drucker's postulate. Therefore softening materials may not achieve steady state behavior. This will affect the EPP procedure but it also affect numerous other ASME code procedures requiring stable cyclic behavior. For example, the conventional code design strategies relies on an elastic analysis and the assumption that the stresses computed for a design cycle will repeat. Notice this means the designer does not need to know in the analysis step the total number of design cycles. For design checks involving creep the designer may need the total design life, for example to enter an isochronous curve, but not the division of that total time into transient and hold times. The designer only needs to know the number of cycles when it comes to doing the actual design calculations. The EPP strain limits method has a similar property: a designer only needs a definition of the appropriate composite cycle. This greatly simplifies design iteration. However, as shown in subsequent chapters, an analysis of a softening structure in general advanced knowledge of both the total design time and the number of design cycles.

\subsection{Bounding the creep/plasticity solution with the cyclic plasticity solution}

The previous section establishes that structures with a certain constitutive response under periodic loading will eventually reach a steady state solution where the stresses and strain rates are periodic. The next key step in the EPP methodology is to bound this periodic creep/plasticity solution with an easy to compute plasticity-only solution. This bound is originally due to Ainsworth [2, 3, 4] for situations above the elastic shakedown limit and Ponter $[21,22]$ below the shakedown limit, with extensions to more complicated constitutive behavior by Carter [10, 9] through a variety of classical techniques [23, 19, 21].

Consider two nearly identical structures: one as defined in Section 2.1 (the creep/plasticity model) and another with the same definition except the creep strain rate is zero, $\dot{\varepsilon}_{c}^{\prime}=0$, and we apply a load multiplier to the cyclic tractions so that $\hat{\boldsymbol{t}}^{\prime}=\lambda \hat{\boldsymbol{t}}$. We call this solution the cyclic plasticity solution and refer to its stress field as $\boldsymbol{\sigma}^{\prime}$, strain field as $\boldsymbol{\varepsilon}^{\prime}$, etc. Note the theorem developed in the previous section applies to the cyclic plasticity model as a special case and both the unmodified and modified problems will eventually converge to some steady cyclic solution. Consider times after both structures achieve this steady state, $t \geq t^{\star \star}$.

Theorem. For times after both structures achieve steady cyclic behavior $t \geq t^{\star \star}$ the incremental creep dissipation of the cyclic plasticity stress field will bound the incremental creep dissipation of the creep/plasticity solution: $\int_{t^{\star \star}}^{t^{\star \star}} \int_{V} D(\boldsymbol{\sigma}) d V d t \leq \int_{\tau} \int_{V} D\left(\boldsymbol{\sigma}^{\prime}\right) d V d t$.

Proof. Consider the incremental virtual work due to the extra load on the cyclic plasticity problem

$$
(\lambda-1) \int_{t^{\star \star}}^{t^{\star \star}+\tau} \int_{\partial \Omega} \hat{\boldsymbol{t}} \cdot\left(\dot{\boldsymbol{u}}^{\prime}-\dot{\boldsymbol{u}}\right) d S d t=\int_{t^{\star \star}}^{t^{\star \star}+\tau} \int_{\Omega}\left(\boldsymbol{\sigma}^{\prime}-\boldsymbol{\sigma}\right):\left(\dot{\boldsymbol{\varepsilon}}^{\prime}-\dot{\boldsymbol{\varepsilon}}\right) d V d t .
$$


Separate this out into components

$$
\int_{t^{\star \star}}^{t^{\star \star}+\tau} \int_{\Omega}\left(\boldsymbol{\sigma}^{\prime}-\boldsymbol{\sigma}\right):\left(\dot{\varepsilon}^{\prime}-\dot{\varepsilon}\right) d V d t=\int_{t^{\star \star}}^{t^{\star \star}+\tau} \int_{\Omega}\left(\boldsymbol{\sigma}^{\prime}-\boldsymbol{\sigma}\right):
$$

By the theorem developed in 2.2 the stress and elastic strains are periodic. Note that the term

$$
\begin{aligned}
\int_{t^{\star \star}}^{t^{\star \star}+\tau} \int_{\Omega} \boldsymbol{\sigma} \dot{\varepsilon}_{e}^{\prime} d V d t=\int_{t^{\star \star}}^{t^{\star \star}+\tau} \int_{\Omega} \boldsymbol{C}: \varepsilon_{e}^{\prime}: \dot{\varepsilon}_{e}^{\prime} d V d t= & \frac{1}{2} \int_{\Omega} \int_{0}^{\tau} \frac{d}{d t}\left[\varepsilon_{e}^{\prime}: \boldsymbol{C}: \boldsymbol{\varepsilon}_{e}^{\prime}\right] d t d V \\
& =\frac{1}{2} \int_{\Omega}\left[\varepsilon_{e}^{\prime}: \boldsymbol{C}: \boldsymbol{\varepsilon}_{e}^{\prime}\right]_{t^{\star \star}}^{t^{\star \star}+\tau} d V=0
\end{aligned}
$$

and that the thermal strain rates are the same in the two structures. Therefore

$$
\int_{t^{\star \star}}^{t^{\star \star}+\tau} \int_{\Omega}\left(\boldsymbol{\sigma}^{\prime}-\boldsymbol{\sigma}\right):\left(\dot{\boldsymbol{\varepsilon}}^{\prime}-\dot{\boldsymbol{\varepsilon}}\right) d V d t=\int_{t^{\star \star}}^{t^{\star \star}+\tau} \int_{\Omega}\left(\boldsymbol{\sigma}^{\prime}-\boldsymbol{\sigma}\right):\left(\dot{\boldsymbol{\varepsilon}}_{p}^{\prime}-\dot{\boldsymbol{\varepsilon}}_{p}-\dot{\boldsymbol{\varepsilon}}_{c}\right) d V d t
$$

Now set the load multiplier to 1 so that the loading on the two problems is identical:

$$
0=\int_{t^{\star \star}}^{t^{\star \star}+\tau} \int_{\Omega}\left[\left(\boldsymbol{\sigma}^{\prime}-\boldsymbol{\sigma}\right):\left(\dot{\varepsilon}_{p}^{\prime}-\dot{\varepsilon}_{p}\right)-\left(\boldsymbol{\sigma}^{\prime}-\boldsymbol{\sigma}\right) \dot{\boldsymbol{\varepsilon}}_{c}\right] d V d t
$$

By Eq. 2.10 the first term is greater or equal to zero, so

$$
\int_{t^{\star \star}}^{t^{\star \star}+\tau} \int_{\Omega}\left(\boldsymbol{\sigma}^{\prime}-\boldsymbol{\sigma}\right) \dot{\varepsilon}_{c} d V d t \geq 0
$$

and we can freely multiply by $n+1$

$$
(n+1) \int_{t^{\star \star}}^{t^{\star \star}+\tau} \int_{\Omega}\left(\boldsymbol{\sigma}^{\prime}-\boldsymbol{\sigma}\right) \dot{\varepsilon}_{c} d V d t \geq 0
$$

Furthermore

$$
\int_{t^{\star \star}}^{t^{\star \star}+\tau} \int_{\Omega} \dot{D}\left(\boldsymbol{\sigma}^{\prime}\right)-\dot{D}(\boldsymbol{\sigma}) d V d t \geq(n+1) \int_{t^{\star \star}}^{t^{\star \star}+\tau} \int_{\Omega}\left(\boldsymbol{\sigma}^{\prime}-\boldsymbol{\sigma}\right): \dot{\boldsymbol{\varepsilon}}_{c} d V d t
$$

by Eq. 2.14. Therefore

or

$$
\int_{t^{\star \star}}^{t^{\star \star}+\tau} \int_{\Omega}\left(\dot{D}^{\prime}-\dot{D}\right) d V d t \geq 0
$$

$$
\begin{aligned}
\int_{t^{\star \star}}^{t^{\star \star}+\tau} \int_{\Omega} \dot{D} d V d t & \leq \int_{t^{\star \star}}^{t^{\star \star}+\tau} \int_{\Omega} \dot{D}^{\prime} d V d t \\
\Delta D & \leq \Delta D^{\prime}
\end{aligned}
$$


This theorem only shows that the total incremental creep dissipation due to the creep/plasticity stress field is bounded by the incremental dissipation of the stress field of the cyclic plasticity solution. Note a subtle but very important point: this bound are applied by first calculating the periodic stress field from the cyclic plasticity solution and then taking that stress field and computing the creep dissipation independently of the original cyclic plasticity problem. There is no direct connection between the strains and deformations in the two problems.

This result is immediately useful when bounding creep damage [10] but not immediately useful for bounding deformations. For bounding the average displacements in the structure we require a bound on the external work $\int_{\partial \Omega} \hat{\boldsymbol{t}} \cdot \boldsymbol{u} d S$.

\subsection{Dissipation bounds to work bounds}

Existing proofs contained in [10] rely on selecting a specific material model to convert the dissipation bound into a bound on the external work of the structure. However, Carter demonstrates the proof for a wide variety of non-unified, creep and plasticity models as well as unified viscoplastic models. In general, these proofs again rely on Drucker's stability postulate, which is not true in general for cyclic softening materials. The simplest case is trivial to prove and so the proof is provided here.

Theorem. For an elastic-creep material without thermal strain the creep dissipation of the cyclic plasticity stress field bounds the mechanical work of the steady cyclic solution.

Proof. The proof is trivial. In the elastic-creep material the strain rate decomposes to

$$
\dot{\varepsilon}=\dot{\varepsilon}_{e}+\dot{\varepsilon}_{c}
$$

By conservation of energy

$$
\Delta W=\int_{\tau} \int_{\partial \Omega} \hat{\boldsymbol{t}} \cdot \dot{\boldsymbol{u}} d S d t=\int_{\tau} \int_{\Omega} \boldsymbol{\sigma}: \dot{\boldsymbol{\varepsilon}} d V d t=\int_{\tau} \int_{\Omega}\left(\boldsymbol{\sigma}: \dot{\boldsymbol{\varepsilon}}_{e}+\boldsymbol{\sigma}: \dot{\boldsymbol{\varepsilon}}_{c}\right) d V d t .
$$

As noted above, the term $\int_{\tau} \int_{\Omega} \boldsymbol{\sigma}: \dot{\boldsymbol{\varepsilon}}_{e} d V d t$ is periodic and so

$$
\Delta W=\int_{\tau} \int_{\Omega} \boldsymbol{\sigma}: \dot{\varepsilon}_{c} d V d t=\Delta D
$$

And so, for a elasticity + creep material

$$
\Delta W \leq \Delta D^{\prime}
$$

This proof did not rely on Drucker's postulate but Carter's proofs for other constitutive forms do. 


\subsection{Bounding the cyclic plasticity solution with an elastic-perfectly plastic solution based on the isochronous curve}

To review, at this stage we have the steady cyclic solution for the creep/plasticity material response and the steady cyclic solution for the cyclic plasticity solution which includes elasticity and plasticity but not creep. We have established that the incremental creep dissipation associated with the cyclic plasticity stress field bounds the incremental external work of the creep/plasticity solution. At this point the bound is only an interesting mathematical relation. It does not directly give a bound on the structural deformation. The final main step in the EPP strain limits bounding method is to bound the creep dissipation of the cyclic plasticity solution with an elastic-perfectly plastic solution based on isochronous curves.

An isochronous curve is the locus of uniaxial stress/strain tuples $(\varepsilon, \sigma)$ reached in some given amount of time $t_{\text {life }}$. At least conceptually these curves can be generated by fitting isochrons through a series of creep tests conducted at different stress levels. In practice long term data is often extrapolated from shorter term tests. These curves are widely used in the ASME design code and have been tabulated for many Division 5 Class A materials [7].

The bound we need to establish is that the incremental mechanical work of an elasticperfectly plastic solution using a pseudo-yield stress set from the isochronous curve at the appropriate design life bounds the creep dissipation associated with the cyclic plasticity solution. Call the EPP solution $\Omega^{\prime \prime}$ and its incremental work $\Delta W^{\prime \prime}$. Then the statement to prove is:

$$
\Delta W \leq \Delta D^{\prime} \leq \Delta W^{\prime \prime}
$$

If this statement holds then the EPP analysis results provide an appropriate bound on the creep/plasticity, steady state solution.

In the original development of the method Carter appeals to an intuitive argument to show that the rapid cycle solution - an optimal cyclic plasticity solution - provides an appropriate bound on the steady cyclic solution [10]. This section attempts to make this connection more rigorously for a highly simplified case.

Consider a 1D, uniaxial geometry with applied strain linearly ramped from zero to $\varepsilon_{f}$ over $t_{\text {life }}$. In one case consider a power law creeping material

$$
\dot{\varepsilon}_{c}=A \sigma^{n} \text {. }
$$

In the second case consider a rigid plastic material with flow stress described by some function $\sigma_{f}(\varepsilon)$. There is no elasticity for both cases.

The stress history of the creeping material will be

$$
\sigma=\left(\frac{\varepsilon}{A t_{\text {life }}}\right)^{1 / n}
$$

during deformation. The total creep dissipation will be

$$
D=\int_{0}^{t_{\text {life }}}\left(\frac{\varepsilon / t_{\text {life }} t}{A t_{\text {life }}}\right)^{1 / n} \frac{\varepsilon}{t_{\text {life }}} d t=\frac{\varepsilon_{f} n}{1+n}\left(\frac{\varepsilon_{f}}{A t_{\text {life }}}\right)^{\frac{1}{n}}
$$


Finally, for this creeping material the expression

$$
\sigma=\left(\frac{\varepsilon}{A t_{\text {life }}}\right)^{1 / n}
$$

describes an isochronous curve for some fixed $t_{\text {life }}$.

The plastic work done by the rigid plastic material over its deformation history is

$$
W=\int_{0}^{\varepsilon} \sigma_{f}(\varepsilon) d \varepsilon .
$$

Now suppose the plastic material's flow curve is the isochronous curve of the creeping material for $t_{\text {life }}$, that is

$$
\sigma_{f}(\varepsilon)=\left(\frac{\varepsilon}{A t_{\text {life }}}\right)^{1 / n} .
$$

Then the plastic work will be

$$
W=\int_{0}^{\varepsilon}\left(\frac{\varepsilon}{A t_{\text {life }}}\right)^{1 / n} d \varepsilon=\frac{\varepsilon_{f} n}{1+n}\left(\frac{\varepsilon_{f}}{A t_{\text {life }}}\right)^{\frac{1}{n}}
$$

And so, for this case the plastic work done by the rigid plastic structure is exactly equivalent to the creep dissipation done by creeping structure

$$
W=D .
$$

This equivalency was only established for a very simple, 1D scenario. If true in the general, 3D context for general constitutive models it could rigorously complete Carter's bounding theorem

$$
\Delta W \leq \Delta D^{\star} \leq \Delta W^{\prime}
$$

showing that the work of a cyclic plasticity solution using the isochronous curve to establish the flow stress bounds the work of a creeping material.

The EPP strain limits method introduces a further simplification. A simplified model might take a rigid plastic formulation with yield stress

$$
\sigma_{Y}=\left(\frac{\varepsilon_{f}}{A t_{\text {life }}}\right)^{1 / n} .
$$

As illustrated graphically in Fig. 2.2, the plastic work of this perfectly-plastic model will bound the plastic work of the model using the creeping material's isochronous curve as the flow stress and so bound the work done by the creeping material. Essentially this is the type of bound used in the EPP strain limits design check. 


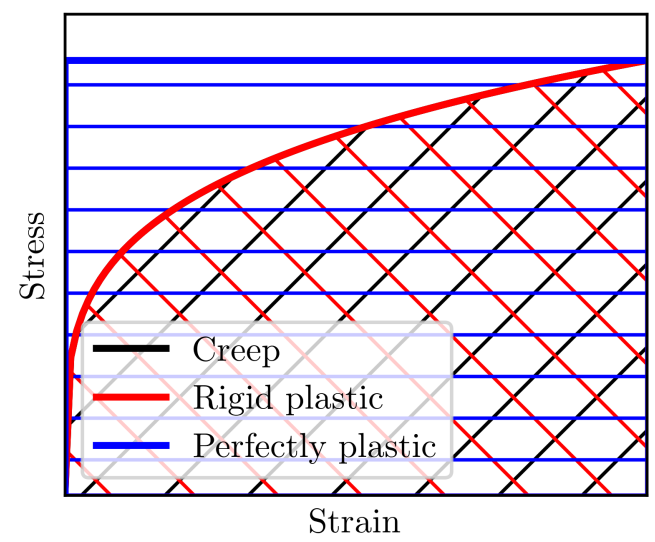

Figure 2.2: A schematic showing how 1) for a simple case the creep dissipation and the work done by an equivalent, rigid plastic model with flow stress given by the isochronous curve is the same and 2) the work of the equivalent plastic case can be bounded by a perfectly plastic material with a yield stress selected to match the value of the isochronous curve at the final strain value.

\subsection{A summary of the EPP method with softening materials}

The derivation above only shows the EPP perfectly-plastic analysis bounds the incremental work of the creep-plasticity solution. However, this bound provides an average bound on the incremental strains and external displacements. Consider the cycle work increment:

$$
\Delta W=\int_{\tau} \int_{\partial \Omega} \hat{\boldsymbol{t}} \cdot \dot{\boldsymbol{u}} d S d t=\int_{\tau} \int_{\Omega} \boldsymbol{\sigma}: \dot{\boldsymbol{\varepsilon}} d V d t
$$

Now suppose we have some bounding solution with the same boundary conditions such that

$$
\Delta W \leq \Delta W^{\prime}
$$

then

$$
\int_{\tau} \int_{\partial \Omega} \hat{\boldsymbol{t}} \cdot \dot{\boldsymbol{u}} d S d t \leq \int_{\tau} \int_{\partial \Omega} \hat{\boldsymbol{t}}^{\prime} \cdot \dot{\boldsymbol{u}}^{\prime} d S d t
$$

but the applied tractions, domain boundary, and period are the same and so

$$
\int_{\tau} \int_{\partial \Omega} \hat{\boldsymbol{t}} \cdot\left(\dot{\boldsymbol{u}}-\dot{\boldsymbol{u}}^{\prime}\right) d S d t \leq 0 .
$$

Therefore, in the sense of an average over a surface, the incremental displacements $\int_{\tau} \dot{\boldsymbol{u}}^{\prime} d t$ bound the incremental displacements $\int_{\tau} \dot{\boldsymbol{u}} d t$. A similar argument could apply to the strains in the bodies. Note the problem setup does not consider the possibility of displacements applied to the domain boundary. This type of boundary condition may appear in practical design situations, for example piping systems impinging on pressure vessels.

The previous sections rederived the EPP strain limits bounding theorem. As noted in the course of the derivation, many of the EPP bounding theorems do not apply to materials that 
do not obey Drucker's postulate. This may mean the bounding theorem fails for softening materials. However, the final derivation showing that a plastic material using an isochronous curve to define the flow stress bounds the work of the corresponding creeping material did not rely on Drucker's postulate. Much of the power the EPP method likely comes from using accurate isochronous curves.

Furthermore, the cyclic bounding theorems certainly can be applied to a certain type of cyclic softening material. Imagine a material that softens with repeated cycling but where the softened flow stress approaches an asymptotic value. Imagine first cycling that material until it reaches its saturated softened state. Because softening has saturated, the future response of the material will be non-softening and the bounding theorems apply to subsequent deformation. Therefore, we might apply the bounding theorems to the response of the material in its saturated state. Figure 1.1 shows that an assumption of saturating hardening is not unreasonable for Gr. 91. This topic is discussed in greater detail below. 



\section{A modeling approach to establishing reduction factors}

The previous chapter demonstrates that, for softening materials, the EPP strain limits method cannot necessarily be shown to be conservative by invoking the standard cyclic plasticity bounding theorems. The key question is then can the method be modified empirically to account for softening behavior.

One approach for modifying the EPP strain limits procedure would be to consider a hypothetical saturated state. Grade 91 softens under cyclic loading. However, imagine that after a certain amount of cycling softening ceases and the material does not continue to lose strength. In this saturated, softened state the material is indistinguishable from a standard material that obeys Drucker's postulate - albeit a material with a potentially very different constitutive response than the original, unsoftened Gr. 91. Conceptually, if the EPP strain limits procedure is applied to this softened, standard material the procedure should produce conservative results. In fact this new method will be somewhat over-conservative, as it assumes a softened material response for the entire loading history when in fact softening will develop gradually with repetitive cyclic load.

This strategy has two prerequisites: 1) that Gr. 91 really does achieve a saturated softening state independent of the strain range of a cycle and 2) that experiments, models, or some combination of the two can provide sufficient information on the softened state of the material to develop design rules for carrying out calculations on the softened state. The next chapter address the first point. This chapter demonstrates that a simple modification of the EPP strain limits method can provide a conservative bound on the response of a structure, given a complete constitutive description of a softening material.

This section represents the response of Gr. 91 using a viscoplastic constitutive model developed by Yaguchi $[30,31]$. Their model represents cyclic softening with a modified Chaboche backstress recovery term (see Fig. 3.1). The original references demonstrate reasonable agreement between the model and the experimental response of Gr. 91 for a variety of loading conditions.

\subsection{A two bar problem}

Figure 3.2 shows a standard two bar problem. The bars have equal length and cross-sectional area $A$. Both share an applied, constant primary load $P$. The first bar undergoes thermal cycling with a temperature/time profile shown in Fig. 3.3. The second bar has constant temperature.

Figure 3.3 shows two temperature cycles. The first, with no hold, is special case of the second, with hold, temperature cycle, i.e. the first case has $t_{\text {hold }}=0$. For an EPP analysis of this geometry both temperature cycles produce the same composite loading cycle. Therefore, an EPP analysis of the two bar structure is the same for any hold time $t_{\text {hold }}$.

Define the total design life of the two bar structure as $t^{\star}$. This design life sets the appropriate isochronous curve. This chapter uses consistent isochronous curves derived from the reference viscoplastic material. These will not necessarily match the the standard ASME code isochronous curves. The EPP strain limits procedure can then be used to ascertain whether the geometry will pass or fail the strain limits design check. This check potentially requires iteration on the target strain $x$. This EPP pass/fail check can be used to compute a design life of the two bar system by iterating on $t^{\star}$ to find the longest $t^{\star}$ that passes the EPP 
FY17 Status Report on the Initial EPP Finite Element Analysis of Grade 91 Steel

July 2017

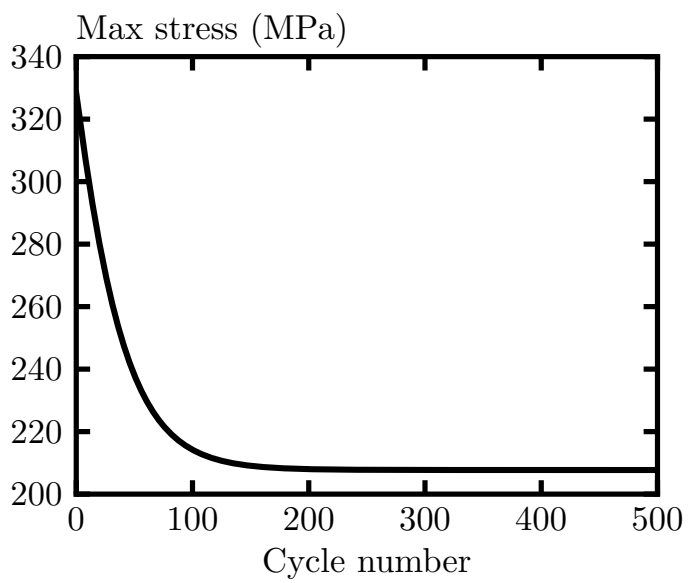

Figure 3.1: Example cyclic softening simulation using the inelastic model. This curve shows the maximum stress versus cycle count for fully-reversed strain controlled loading with $\Delta \varepsilon=$ 0.01 at $T=600^{\circ} \mathrm{C}$ - the maximum cycle temperature. The model captures cyclic softening and predicts a saturating softening response - eventually the processes causing softening saturate and the material achieves a constant flow stress.

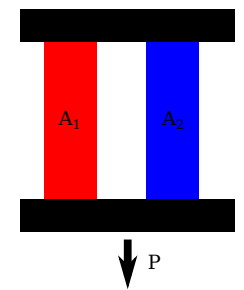

Figure 3.2: Example two bar geometry.

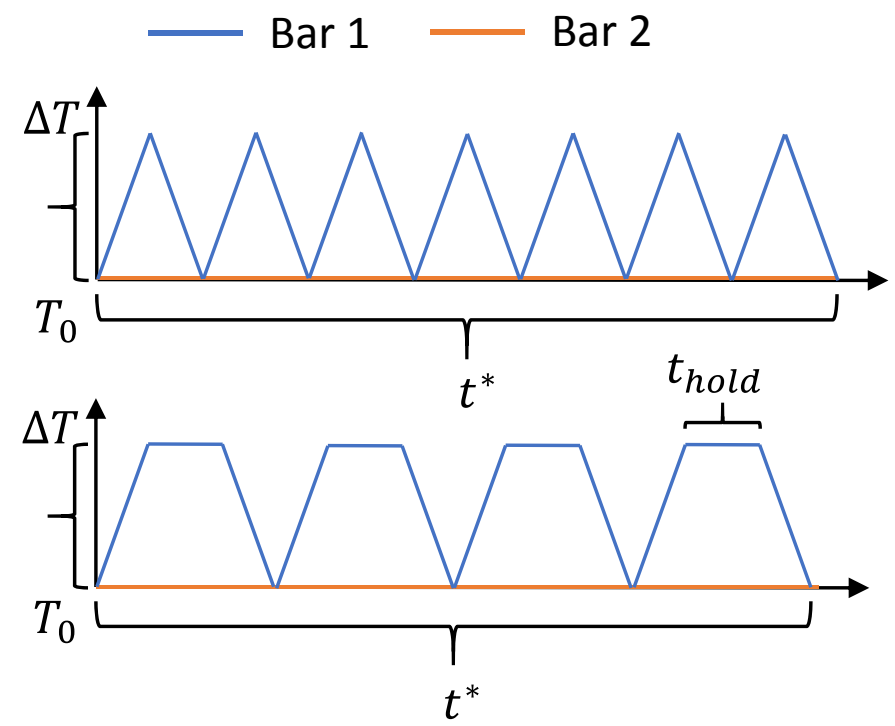

Figure 3.3: Two bar temperature cycles. The top, no-hold cycle is a special case of the generic cycle on the bottom of the figure. 
FY17 Status Report on the Initial EPP Finite Element Analysis of Grade 91 Steel

July 2017

\begin{tabular}{lll}
\hline Property & Description & Value \\
\hline$A$ & Bar cross-sectional area & $126 \mathrm{~mm}^{2}$ \\
$P$ & Primary load & $50.4 \mathrm{kN}$ \\
$T_{0}$ & Reference temperature & $200^{\circ} \mathrm{C}$ \\
$\Delta T$ & Maximum bar temperature difference & $400^{\circ} \mathrm{C}$ \\
$\dot{T}$ & Heating/cooling rate & $0.5^{\circ} \mathrm{C} / \mathrm{s}$ \\
\hline
\end{tabular}

Table 3.1: Two bar problem example loading parameters.

\begin{tabular}{lll}
\hline$t_{\text {hold }}$ & Design life & Number of cycles to $1 \%$ strain \\
\hline $0 \mathrm{hrs}$ & $37 \mathrm{hrs}$ & 84 \\
$1 \mathrm{hrs}$ & $116 \mathrm{hrs}$ & 80 \\
$10 \mathrm{hrs}$ & $756 \mathrm{hrs}$ & 72 \\
$100 \mathrm{hrs}$ & $8,880 \mathrm{hrs}$ & 88 \\
$1000 \mathrm{hrs}$ & $102,600 \mathrm{hrs}$ & 102 \\
\hline
\end{tabular}

Table 3.2: Design lives computed via inelastic analysis, for different values of $t_{\text {hold }}$.

strain limits criteria. This procedure was carried out for the loading parameters described in Table 3.1. It arrives at a life for the two bar system of $245 \mathrm{hrs}$. Note this EPP design life, using the standard, code case procedure, is completely independent of the actual number of cycles the system will experience.

Alternatively, design lives for this system can be calculated directly from the inelastic model. This requires selecting a heating and cooling rate, listed in Table 3.1 for this particular example, and a $t_{\text {hold }}$. Selecting these fully defines the load cycle. Then a simulation of the two bar system can be run, repeating the temperature cycle until the one of the two bars exceeds $1 \%$ inelastic strain. The number of cycles until $1 \%$ strain multiplied by the cycle period $\left(\tau=2 \Delta T / \dot{T}+t_{\text {hold }}\right)$ is the design life of the system for that particular hold time. This processes was repeated several times for different values of $t_{\text {hold }}$. Table 3.2 summarizes the results of these calculations.

For shorter values of hold time the two bar system undergoes relatively more cycling than holding. This generates a relatively larger amount of cyclic softening in the material, which in turn causes more creep strain per cycle and produces shorter design lives. The first chapter of this report summarizes the causes of softening behavior of Gr. 91.

Because the number of cycles a structure experiences does not influence unmodified EPP strain limits analysis, the lifetime computed from this procedure can be compared to the lifetimes computed using the inelastic model. Figure 3.4 summarizes this comparison by plotting hold time $t_{\text {hold }}$ versus the ratio $t_{\text {life }}^{\text {inelastic }} / t_{\text {life }}^{E P P}$. The data shows that EPP is not conservative, that is the inelastic model predicts a shorter design life than the EPP strain limits procedure, for low values of $t_{\text {hold }}$. This is the region where cyclic softening has the most effect. As Chapter 2 demonstrates, the EPP methodology may not be conservative indeed the EPP bounds do not apply - for softening materials. However, the EPP strain limits method can be corrected to account for softening and regain conservatism for design cases including high numbers of cycles. 


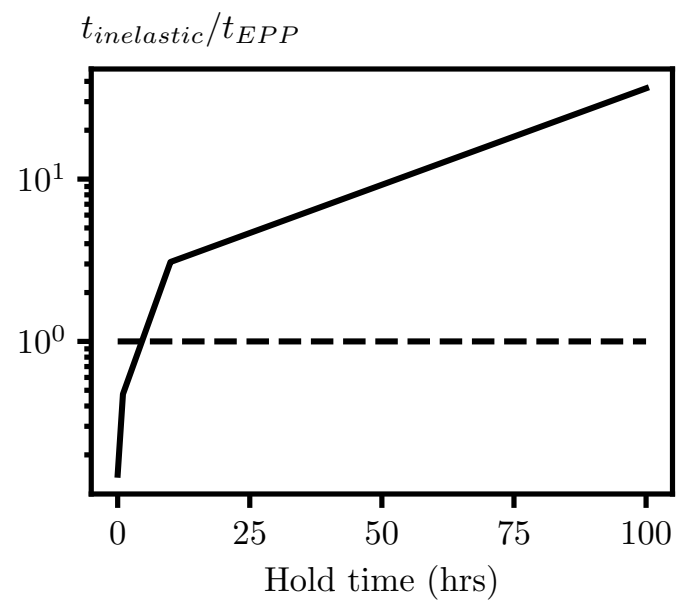

Figure 3.4: Plot illustrating the region the conservatism and non-conservatism of the unmodified EPP procedure versus inelastic simulation results. The dashed line indicates the tradeoff condition - below this line, in the region of high cyclic softening - the unmodified strain limits procedure is not conservative.

\subsection{Simulating softened isochronous curves}

A direct modification of the EPP strain limits methodology could use softened isochronous curves in place of isochronous curves developed from the initial state of the material. Isochronous curves can be generated from a series of creep tests at different stress levels or, for an inelastic constitutive model simulations of creep tests at different stresses. A general procedures is to collect a series of creep data as strain versus time histories. An isochronous stress/strain curve is simply an isochron through that data - the locus of $(\varepsilon, \sigma)$ points all at a given time $t^{\star}$.

The isochronous curves for Gr. 91 in the ASME design code and used in the unmodified EPP calculations above start the creep tests/simulations with the initial, unsoftened material state. These curves then do not capture any effect of cyclic softening. In fact, a prior history of strain-controlled cycling will increase the creep rate of the sample, as compared to a sample that did not undergo cycling (see Fig. 4.1). One way to account for cyclic softening in the isochronous curves and therefore in the EPP methodology would be to generate a series of softened isochronous curves. This would require a series of creep tests or simulations of creep tests starting from a cycled, softened material state. Ideally this procedure would be repeated for a variety of cycle types - strain ranges and number of cycles - to develop a complete description of the effect of cycling on the creep behavior of the material.

This procedure is not practical experimentally as it requires a very large set of long term creep tests. However, it is practical to simulate. Figure 3.5 illustrates one series of softened isochronous curves, simulating the material response with the inelastic constitutive model described above. This series of curves was created by first cycling the material $N$ times at $600^{\circ} \mathrm{C}$ under fully-reversed, strain controlled loading with a total strain range of $1 \%$. A similar and complete series of curves was generated for temperatures in between $200^{\circ} \mathrm{C}$ and $600^{\circ} \mathrm{C}$ - the temperature range used in the sample two bar problem. This series of 
curves did not consider the effect of strain range but only the effect of cycle count. The strain range effect was neglected because, for this particular inelastic model, cyclic softening 1) develops proportional to the total equivalent plastic strain and 2) eventually saturates. Therefore, provided the strain controlled cyclic loading exceeds the yield stress, there will be an equivalence between the strain range and the number of cycles such that it is possible to match an arbitrary combination strain range and cycle count to the $1 \%$ strain range curves generated here. If the cyclic loading does not exceed the yield stress the material will not soften. The effect of strain range will need to be quantified experimentally on the actual material. However, it is not unreasonable to expect it to be a function of equivalent plastic strain, as the softening relaxation mechanism is likely dislocation related.

Given these softened curves, we could propose a modified EPP strain limits procedure. This procedure would follow the same steps as the unmodified code case procedure except the designer would be required to determine the expected number of design cycles the component will undergo in its design life, estimate the maximum strain range this cycle will cause a material point to undergo, use this information to look up the appropriate softened isochronous curve, and use this softened curve in place of the standard, unsoftened curve when computing the EPP pseudo-yield stresses. The strain limits code case specifies the pseudo-yield stress as the smaller of a value selected from the isochronous curve and the material $S_{y}$ so a similar procedure will be required to establish the softened material yield stress.

This potential modification has two flaws:

1. It requires generating and listing in the code a large series of softened isochronous curves.

2. It requires an estimation of the strain range and number of cycles a component will undergo in service.

The next section describes a method for avoiding requiring the code to specify a large number of softened isochronous curves. A later section addresses the second flaw in this list, proposing a procedure to eliminate any consideration of cycle count and cycle strain range by referencing a saturated, softened state for the material.

\subsection{Calculating softening factors}

Rather than providing a complete set of softened curves a simplified approach could provide tabulated softening reduction factors to apply to the existing isochronous curves. The approximate, softened isochronous curve value would be the value at the appropriate strain from the current cuve multiplied by this softening factor.

This approach is only exact if the softened isochronous curves are simple reductions of the original, unsoftened curves, i.e.

$$
\sigma_{\text {soft }}(\varepsilon)=f_{\text {soft }} \sigma_{\text {iso }}(\varepsilon)
$$

where $\sigma_{\text {soft }}(\varepsilon)$ is the softened isochronous curve, $f_{\text {soft }}$ is the softening factor, and $\sigma_{\text {iso }}$ is the unsoftened isochronous curve. In general this will not be the case. We might consider two 
FY17 Status Report on the Initial EPP Finite Element Analysis of Grade 91 Steel July 2017

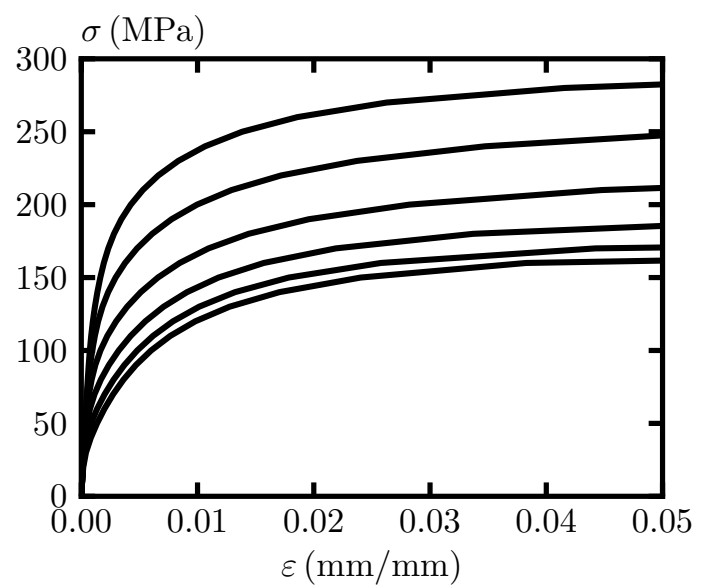

(a) $N=0$

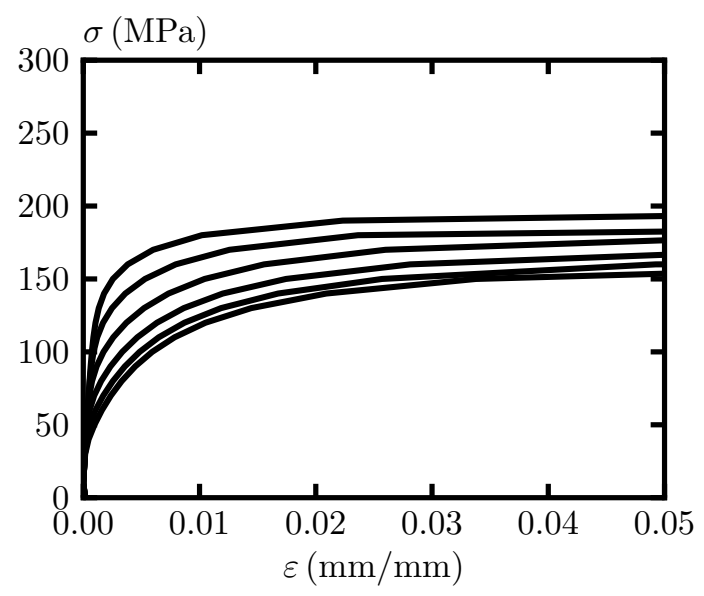

(c) $N=100$

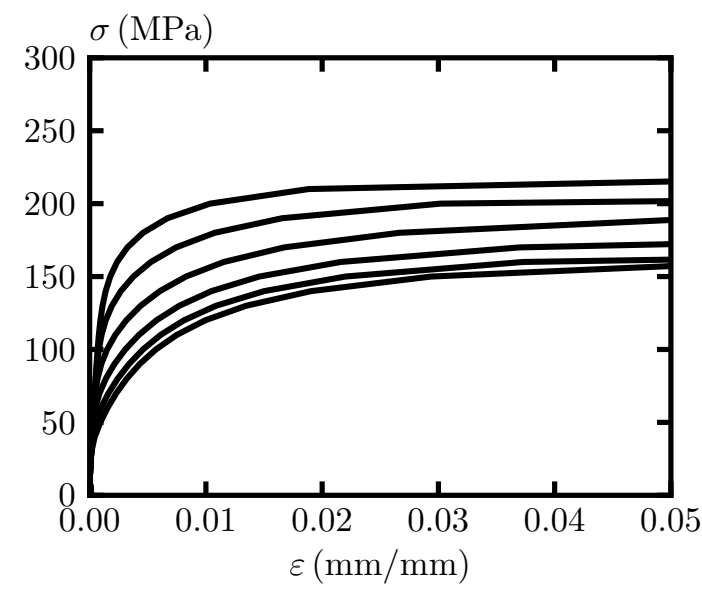

(b) $N=50$

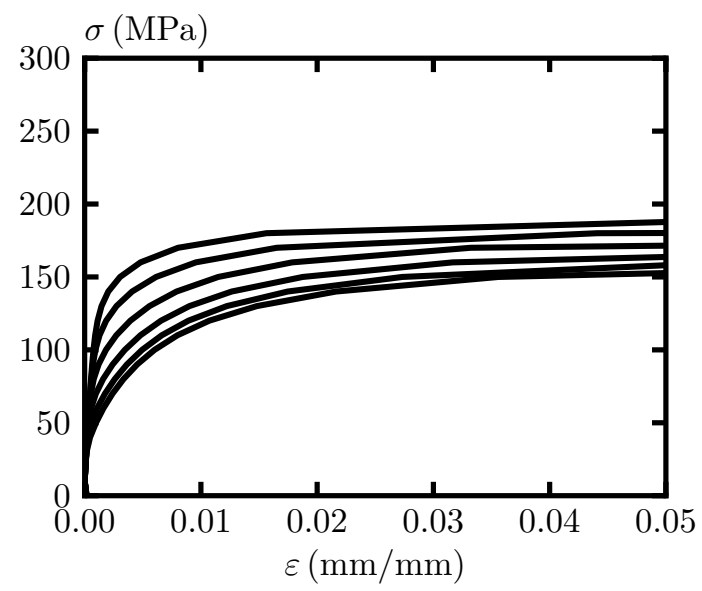

(d) $N=500$

Figure 3.5: Softened isochronous curves computed for the Gr. 91 inelastic model using a cycle strain range of $\Delta \varepsilon=1 \%$ at a temperature of $600^{\circ} \mathrm{C}$. The subfigure captions give the number of cycles. From the top of each plot to the bottom the lines represent isochrons of 1 hour, 10 hours, 100 hours, 1000 hours, 10000 hours, and 100000 hours. 
approaches for generating approximate factors. The first averages the ratio of the softened and unsoftened curves

$$
f_{\text {soft }}^{(a v g)}=\int_{0}^{\varepsilon_{f}} \frac{\sigma_{\text {soft }}}{\sigma_{\text {iso }}} d \varepsilon / \varepsilon_{f}
$$

and the second uses the exact reduction factor for some strain value

$$
f_{\text {soft }}^{(\text {avg })}=\frac{\sigma_{\text {soft }}\left(\varepsilon_{0}\right)}{\sigma_{\text {iso }}\left(\varepsilon_{0}\right)} .
$$

This report adopts the second approach, using $\varepsilon_{0}=1 \%$, inelastic strain, as the reference strain, corresponding to the initial value of the target strain in the existing EPP strain limits code case procedure. Furthermore, rather than compute a correction factor for each design life, the correction factors used here average the individual correction factors for all design lives at the same temperature. Therefore, the final correction is a function of temperature and number of cycles only.

The interpolation

$$
\begin{aligned}
f_{\text {soft }}(N, T)=\left[8.943 \times 10^{-7} \exp (0.01964 T)+0.1655\right] & \\
& {\left[\exp \left(-\left\{2.821 \times 10^{-5} T-1.235 \times 10^{-3}\right\} N\right)-1\right]+1 }
\end{aligned}
$$

for $200^{\circ} \mathrm{C} \leq T \leq 600^{\circ} \mathrm{C}$ approximates the softening factors derived from the inelastic model isochronous curves. This interpolation equation obeys two trends observed in the raw data:

1. The correction factor decreases with increasing temperature.

2. The correction factor decreases with increasing numbers of cycles but eventually saturates to some asymptotic value.

The second observation is critical and is caused by the saturation of cyclic softening in the Yaguchi model (c.f. Fig. 3.1). If the true material softening does not saturate neither will the isochronous softening factors.

For reference, Fig. 3.6 plots the softening factor as a function of temperature for $N=100$ and Fig. 3.6 plots the factor as a function of cycle count for $T=550^{\circ} \mathrm{C}$.

\subsection{A modified EPP strain limits procedure}

Two modifications to the EPP strain limits procedure are possible starting from the softening factor interpolation formula (Eq. 3.4). The first procedure is less over-conservative but requires an estimation of the strain range at each material point and the number of actual load cycles the structure will undergo. The second procedure is more conservative, does not require a strain range or the true number of cycles, but does assume a saturating softening response.

\subsubsection{Procedure requiring strain range and cycle count}

1. Determine a design life $t_{\text {life }}$ and define a composite cycle following the existing strain limits code case procedure. 


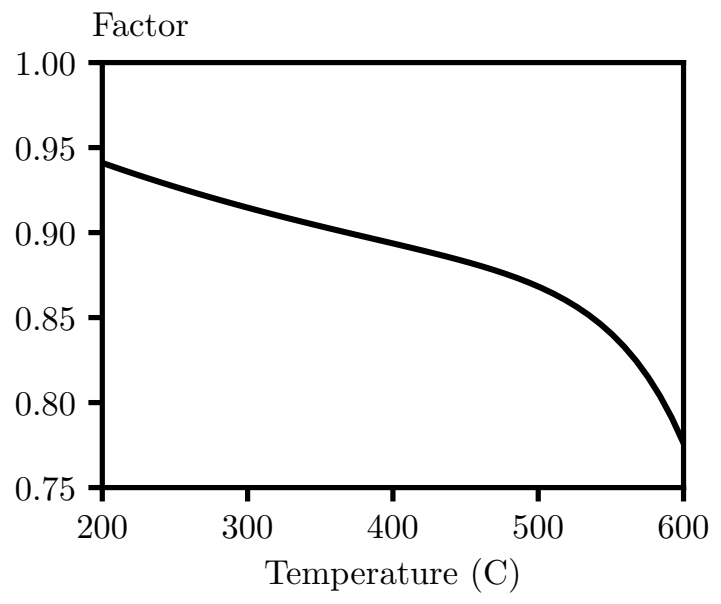

(a) Effect of temperature at $N=100$ cycles.

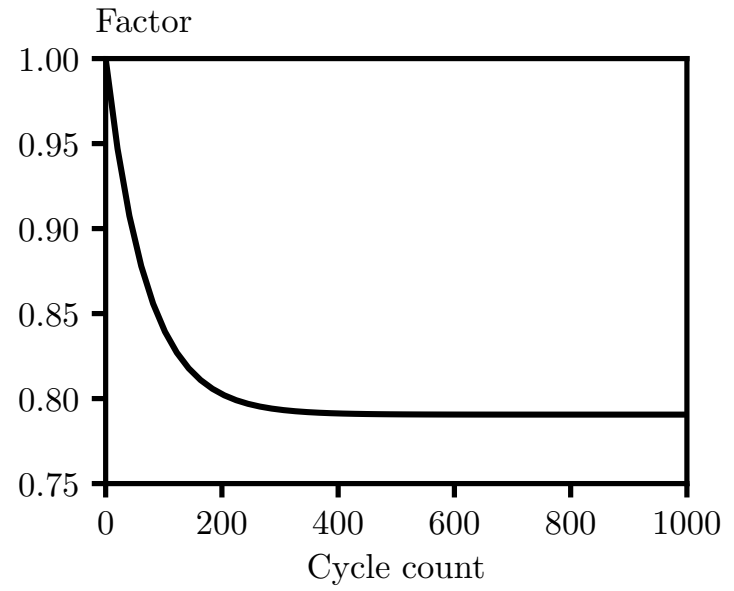

(b) Effect of cycle count at $T=550^{\circ} \mathrm{C}$.

Figure 3.6: Plots illustrating general trends in the softening reduction factors.

2. Estimate the maximum strain range each material point experiences in service, $\Delta \varepsilon_{i}$.

3. Determine the actual number of service cycles the component will experience in its design life.

4. Complete the existing EPP strain limits procedure but replace the temperature-dependent pseudo-yield stress $S_{x T}$ with the pseudo-yield stress $f_{\text {soft }}\left(N, \Delta \varepsilon_{i}, T\right) S_{x T}$.

This report does not consider the effect of strain range, additional calculations or experiments would be required to establish the softening factor $f_{\text {soft }}\left(N, \Delta \varepsilon_{i}, T\right.$ ) (but see the note on the equivalent of cycles and strain range above). These simulations or experiments would be fatigue-creep with the initial fatigue loading now considering different numbers of cycles, different strain ranges, and different temperatures. Furthermore, there is no straightforward way to complete step 2 of this modified procedure as it strictly requires a complete inelastic analysis of the structure, eliminating one main advantage of the EPP method. A possibility would be to use the strain range at each material point for the steady state EPP analysis. Additional work would be required to determine whether this strain range appropriately bounds the strain range in actual engineering structures. Furthermore, using the EPP strain range would introduce a second iterative step into the analysis procedure. The pseudo-yield stress used in the EPP analysis would be a function of the strain range found by that analysis, requiring multiple iterations of changing the $\Delta \varepsilon_{i}$ used to compute the softening factor until it matched the actual strain range computed in the EPP analysis. Fixed point iteration could be used to converge to the correct strain range but it does complicate the EPP strain limits procedure.

\subsubsection{Simplified procedure requiring saturating softening behavior}

As observed above, the softening factors computed from the inelastic simulation results converge to some asymptotic value as the cycle count increases. Table 3.3 tabulates these 


\begin{tabular}{cc}
\hline Temperature & Factor \\
\hline $200^{\circ} \mathrm{C}$ & 0.83 \\
$300^{\circ} \mathrm{C}$ & 0.83 \\
$400^{\circ} \mathrm{C}$ & 0.83 \\
$500^{\circ} \mathrm{C}$ & 0.82 \\
$600^{\circ} \mathrm{C}$ & 0.72 \\
\hline
\end{tabular}

Table 3.3: Saturated softening factors computed from the inelastic numerical experiments.

saturated softening factors at different temperatures.

These saturated softening factors suggest a modification to the EPP strain limits method to handle cyclic softening that does not require knowing either the strain range or actual number of design cycles. The modification is simple: follow the existing code case procedure but replace the temperature dependent pseudo-yield stress $S_{x T}$ with the softened pseudoyield stress $f_{\text {soft }}(T) S_{x T}$ where $f_{\text {soft }}(T)$ is the saturated softening factor, for example as tabulated in Table 3.3.

This procedure retains the full simplicity of analysis of the existing EPP strain limits methodology, not requiring knowledge of the true number of service cycles the component will undergo nor the strain range at each material point. It will be more conservative than the detailed procedure described above and it assumes and requires that the material experience saturating work softening.

Following this modified EPP strain limits procedure produces an EPP design life for the example two bar problem of $0 \mathrm{hrs}$ - that is the EPP analysis fails to shakedown for any target strain $x$ in the range $0 \% \leq x \leq 1 \%$. This is a conservative prediction of the design life of the structure as computed by inelastic analysis for the shortest $t_{\text {hold }}$ and highest number of actual service cycles, which was $37 \mathrm{hrs}$. This procedure can be over-conservative for cases with long hold time, relative to the number of cycles. Like the baseline EPP method it predicts a single life for the two bar system, completely disregarding the actual number of cycles and holds the system undergoes. This means it will produce a design life reflecting the worst case, most cycles loading. This life will be very conservative compared to longest hold, least cycles life, which was 102,600 hrs here.

This procedure for cyclic softening is a simple modification of the original EPP strain limits code case and retains all the advantages of the original procedures. As such, the authors would recommend it, over the detailed procedure in the previous subsection, to form the basis of a code case for EPP analysis with Gr. 91 steel. However, the modified procedure requires saturating softening behavior and an interpolation formula or tabulation of the saturated softening factors, both of which should be established experimentally.

If less over-conservatism is desired a third procedure could consider a "half softened" state as the reference from which to calculate softened isochronous curves and the EPP pseudoyield stress. This could be defined by assuming a saturating material response, deriving a cyclic stress/strain curve for the material, and defining the reference state as the material state at a point along the cyclic stress/strain curve between the initial cyclic flow stress and before the material softening saturates. Further study would be required to assess this approach to ensure it is not under-conservative and to identify a reasonable reference point 
FY17 Status Report on the Initial EPP Finite Element Analysis of Grade 91 Steel July 2017

along the cyclic stress/strain curve. 


\section{Experimental reduction factors}

The previous chapter established a methodology for modifying the EPP strain limits procedure to account for cyclic softening. The method has two prerequisites: the cyclic softening response of the material must saturate and a factor, as a function of temperature, must describe the saturated, softened isochronous curve. This section describes testing these prerequisites against experimental data.

\subsection{Proposed experiment type}

Ideally, a complete set of softened isochronous curves could be generated experimentally through a large series of fatigue/creep tests. For each specimen the test protocol would be:

1. Cycle the specimen under symmetric strain controlled loading at temperature $T$ for $N$ cycles with a total strain range of $\Delta \varepsilon$.

2. Unload the specimen. Reload to stress $\sigma$ and creep the specimen to failure, recording the creep curve $\varepsilon$ as a function of time.

Each softened isochronous curve describes creep in the material for a set $T, N$, and $\Delta \varepsilon$. Working from the set of specimens with these parameters fixed the isochronous curve for design life $t_{\text {life }}$ is the locus of points $(\varepsilon, \sigma)$ such that $\varepsilon=\varepsilon\left(t_{\text {life }}\right)$ on the creep curve with hold stress $\sigma$. The softening factors could be computed directly from this hypothetical set of experimentally-measured softened isochronous curves.

This procedure would require a vast number of experiments including a large number of long term creep tests. As such it may not be practical. A simplified test procedure would consider only the instantaneous acceleration of the creep rate associated with some amount of cycling. These tests can only give an estimate of the true softening factor but for a fixed $T$ and $\Delta \varepsilon$ a single experiment produces data for all cycles counts and, with an approximation, stresses, vastly reducing the number of experiments required to establish the softening factors.

The simplified test procedure uses a single specimen to measure the instantaneous increase in creep rate due to cycling. For a selected temperature and strain range the experimental protocol is:

1. Choose a hold stress value $\sigma$ using the existing, unsoftened material data so that significant creep will occur in a short period of time $t_{\text {hold }}$.

2. Cycle the specimen in strain control for $n<N$ cycles at temperature $T$ and strain range $\Delta \varepsilon$.

3. Unload the specimen and switch to load control. Load the specimen to the selected hold stress and hold fort $t_{\text {hold }}$. Record the creep strain versus time data.

4. Repeat steps 2 and 3 until the specimen fails.

This procedure produces a series of accelerated creep curves at $N=n, 2 n, 3 n$, etc. Figure 4.1 plots the strain versus time history for one test with $T=600^{\circ} \mathrm{C}, \Delta \varepsilon=1 \%, \sigma=150 \mathrm{MPa}$, 


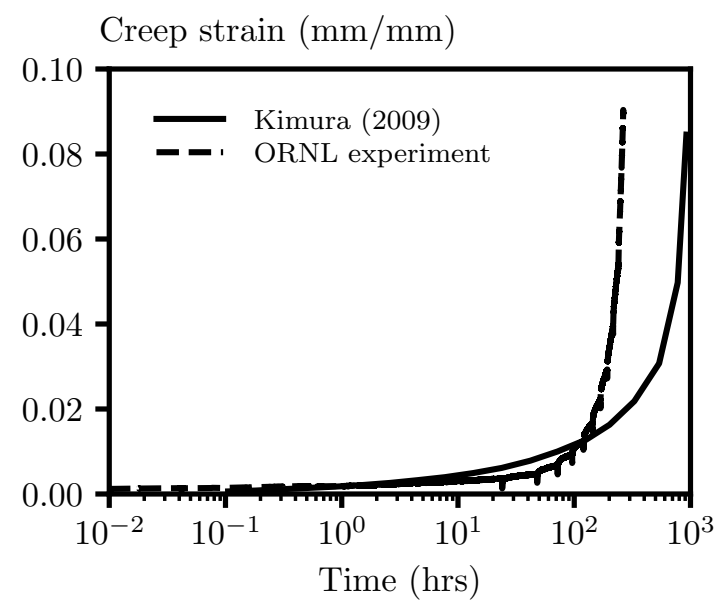

Figure 4.1: Data collected from fatigue/creep tests. The softened curve is from ORNL experimental work while the reference curve is from [18].

$t_{\text {hold }}=24 \mathrm{hrs}$, and $n=100$ along with a reference creep curve with no cycling at the same temperature and hold stress. This experimental series can be logically divided into 11 accelerated creep curves. This series of accelerated creep curves together with an uncycled reference creep curve, ideally from the same batch of material, provides sufficient information to approximate the softening reduction factor at each number of cycles.

The figure shows how repeated cycling softens the material and increases the creep rate relative to the reference curve on unsoftened material. The next section translates this experimental data into approximate isochronous softening factors.

\subsection{Calculating approximate softening factors from experimental data}

For each creep segment (i.e. the hold in between cycling) in an experiment like the one shown in Fig. 4.1 the average acceleration of the creep rate over the segment can be translated into an approximate isochronous curve softening factor. To do this assume a constant creep rate over each creep segment of the fatigue/creep experiment. Call the average creep rate in each segment $\dot{\varepsilon}_{i}^{(e x p)}$ and let the times bounding this region of the creep curve be $t_{i}^{(\text {start })}$ and $t_{i}^{(e n d)}$. Strictly, when computing times the time required to cycle the specimen should be neglected and only the time at the hold stress counted. However, the cycle time is short compared to the creep time in these experiments. Next compute the average creep rate in the reference, uncycled experiment $\dot{\varepsilon}_{i}^{(\text {ref })}$ for the same time interval $\left[t_{i}^{(\text {start })}, t_{i}^{(\text {end })}\right]$. Define the creep acceleration factor

$$
a_{i}=\frac{\dot{\varepsilon}_{i}^{(e x p)}}{\dot{\varepsilon}_{i}^{(r e f)}} .
$$

To compute an isochronous stress/strain softening factor first imagine a hypothetical set of creep tests used to generate isochronous curves where the creep rate for each test is constant though the constants rates vary with the applied stress and test temperature. Furthermore, ignore the initial strain present in the material due to elasticity and plasticity 
from applying the hold stress. Describe each test by the creep law

$$
\varepsilon_{\text {creep }}=A t
$$

where $A$ is the creep rate. At a constant temperature each different value of hold stress will produce a different creep rate. Describe this variation with the function $A(\sigma)$ where $\sigma$ is the hold stress. Each isochronous curve at a given $t_{\text {life }}$ is the solution to the equation

$$
\varepsilon=A(\sigma) t_{\text {life }} .
$$

This expression gives the strain as a function of stress. The more usual expression is the inverse

$$
\sigma=A^{-1}\left(\frac{\varepsilon}{t_{\text {life }}}\right) .
$$

Now scale the creep rate $A$ by some factor $a_{i}$ such that the new creep/time equation is

$$
\varepsilon_{\text {creep }}=a_{i} A t
$$

The resulting isochronous curve is

$$
\sigma=A^{-1}\left(\frac{\varepsilon}{a_{i} t_{l i f e}}\right)
$$

Given a reference set of isochronous curves described by $A^{-1}\left(\varepsilon, t_{l i f e}\right)$ the approximate softened isochronous curve will be described by the function $A^{-1}\left(\varepsilon, t_{l i f e} a_{i}\right)$. That is, using the reference curve for some design life $t_{\text {life }}$ the softened curve for $t_{\text {life }}$ will be the reference curve for design life $a_{i} t_{l i f e}$. The cyclic softening factor can be computed from the reference, unsoftened curve and the approximate softened curve using one of the two procedures described above. Figure 4.2 illustrates computing the softening factors for 100 hours design life at $600^{\circ} \mathrm{C}$ using the experimental data summarized in Fig. 4.1 and the ASME Gr. 91 isochronous curves to describe the reference, unsoftened material response. Tables 4.1 and 4.2 compare the calculated softening factors from experimental data to the model-based factors derived above for 100, 1000, and 10000 hour lives for $600^{\circ}$ and $550^{\circ} \mathrm{C}$.

The tables show the modeling assumption of having all isochronous curves at a given temperature share the same softening factor is justified. For a given number of cycles the experimental factors for the three considered design lives are very similar. For the number of cycles considered here the model-based and experimental softening factors are similar. The model softens somewhat more quickly than the experimental material and eventually the softening in the model saturates. The experiments did not observe saturating softening at this temperature.

\subsection{Saturating softening}

Figure 4.3 reproduces Fig. 1.1 from the introduction and shows the experimental cyclic softening behavior of Gr. 91 at two different temperatures. This figure adds annotations splitting the softening behavior into regions. Notice neither the high temperature nor the low temperature experiments shows saturating softening. However, as the figures indicate, the final 


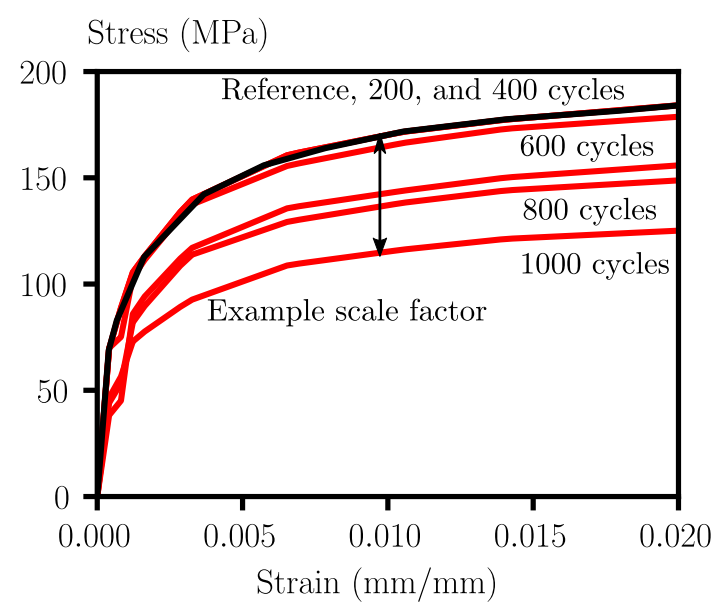

Figure 4.2: Plot showing the scaled isochronous curves, using the procedure described in this section, the ORNL experimental data, and the ASME isochronous reference curves. The figure shows the softened curves for 200, 400, 600, 800, and 1000 cycles, in addition to the reference curve. The arrow on the diagram shows how a softening factor can be computed from this data, using the $1 \%$ strain method developed above. The kinks in the softened curves is interpolation error. The ASME code only provides curves for a finite set of design lives, requiring interpolation to lives in between these values.

\begin{tabular}{ccccc}
\hline & & \multicolumn{3}{c}{ Experimental } \\
Cycle & Model & $100 \mathrm{hrs}$ & $1000 \mathrm{hrs}$ & $10000 \mathrm{hrs}$ \\
\hline 0 & 1.0 & 1.0 & 1.0 & 1.0 \\
200 & 0.73 & 1.0 & 1.0 & 1.0 \\
400 & 0.72 & 0.97 & 0.96 & 0.96 \\
600 & 0.72 & 0.84 & 0.87 & 0.86 \\
800 & 0.72 & 0.81 & 0.79 & 0.75 \\
1000 & 0.72 & 0.68 & 0.66 & 0.68 \\
\hline
\end{tabular}

Table 4.1: Table comparing the experimental softening factors to the model-based softening factors for $600^{\circ} \mathrm{C}, 100,1000$, and 10000 hours life, and various numbers of cycles. The experimental factors were computed from ORNL data for the softened case and reference creep data sourced from [18].

\begin{tabular}{ccccc}
\hline & & \multicolumn{3}{c}{ Experimental } \\
Cycle & Model & $100 \mathrm{hrs}$ & $1000 \mathrm{hrs}$ & $10000 \mathrm{hrs}$ \\
\hline 0 & 1.0 & 1.0 & 1.0 & 1.0 \\
100 & 0.84 & 0.99 & 0.99 & 0.99 \\
200 & 0.80 & 0.92 & 0.90 & 0.89 \\
\hline
\end{tabular}

Table 4.2: Table comparing the experimental softening factors to the model-based softening factors for $550^{\circ} \mathrm{C}, 100,1000$, and 10000 hours life, and various numbers of cycles. The experimental factors were computed from ORNL data for the softened case and reference creep data sourced from [18] 


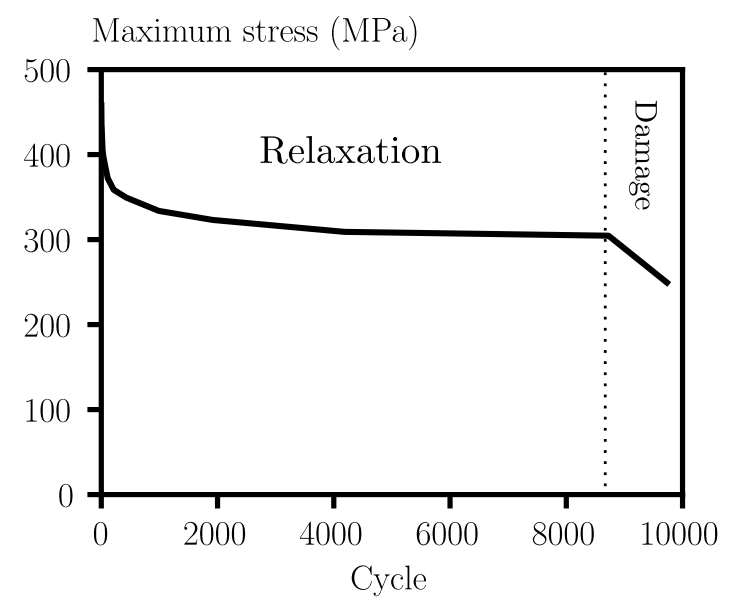

(a) $\mathrm{T}=320^{\circ} \mathrm{C}$ and $\Delta \varepsilon=0.7 \%[17]$.

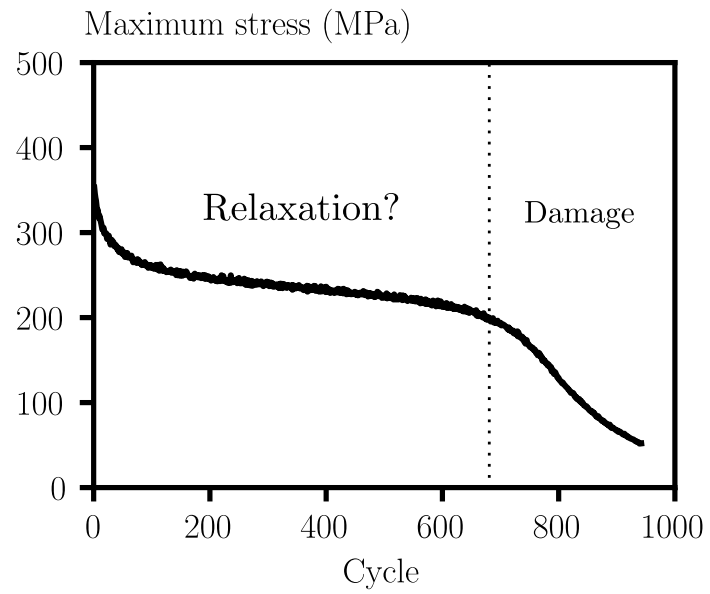

(b) $T=600^{\circ} \mathrm{C}$ and $\Delta \varepsilon=1 \%$ (personal communication with M. Li, Argonne National Laboratory).

Figure 4.3: Plots illustrating G. 91 cyclic softening behavior at low and high temperature.

downturn in the experimental softening curve is most likely due to damage, rather than microstructural relaxation processes causing softening. The ASME code guards against creepfatigue damage through a separate design check which should prevent designs from experiencing any significant creep-fatigue damage. So for the purposes of establishing isochronous softening factors for EPP strain limits procedure we can ignore the terminal softening due to damage. Note that the existing EPP creep-fatigue code case again assumes a cyclic hardening material, so additional work may be required to modify the procedure to prevent creep-fatigue damage in Gr. 91 and other similar softening materials. Furthermore, while attributing the final downswing in the softening curves to damage is reasonable additional experimental work should rigorously link damage accumulation to the region of increased softening. Interrupted tests coupled with microscopy to look for void and microcracks could make this connection.

There is a significant difference between the higher and lower temperature softening data. Neglecting the damaged region, the low temperature softening response seems to saturate. The high temperature data does not seem to saturate. Softening in Gr. 91 is often attributed to microstructural rearrangement, principally relaxation of the dislocation structure but also the migration of carbides and the growth of sub-grain boundaries [1, 15, 24]. These processes are typically thought of as saturating as, for given thermomechanical conditions, they represent the microstructural moving towards some new equilibrium state. There could be two interpretations of the non-saturating but possibly undamaged region of the high temperature softening curve. One interpretation is this region is actually a saturating process with a long characteristic time scale - so long that damage begins to develop in the material before it reaches its saturated state. The second interpretation is that the non-saturating softening is actually some early-time damage process. Tests coupled with microscropy could determine which of these two theories is correct.

If the softening is caused by some saturating process with a long time scale one way to ap- 
FY17 Status Report on the Initial EPP Finite Element Analysis of Grade 91 Steel

July 2017

ply the modified EPP procedure developed here could be to extrapolate the experimentallyderived softening factors out to saturation, neglecting the higher cycle data on clearly damaged material. However, if the softening is caused by some early time damage process this extrapolation would be inappropriate. Further work is required to ascertain which behavior is actually occurring in the material. 


\section{$5 \quad$ Grade 91 two bar tests}

\section{$5.1 \quad$ Test setup}

The previous sections establish a modified EPP strain limits procedure designed to account for cyclic softening. This section tests the modified procedure against a series of two bar tests to assess its conservatism.

The two bar geometry is the same as used in the numerical simulations above, shown in Fig. 3.2. The temperature cycle is different: both bars undergo thermal cycling. However the test delays the heating of one bar relative to another, introducing a cyclic thermal that can cause ratcheting. Figure 5.1 illustrates the experimental thermal profile.

A series of tests was completed at ORNL on Gr. 91 samples. A parallel report details the experimental conditions, setup, and results. The experimental temperature range was $T_{a}=350^{\circ} \mathrm{C}$ to $T_{b}=650^{\circ} \mathrm{C}$. Table 5.1 summarizes the result of the two bar tests. The steady state, cyclic ratcheting strain accumulation in the two bar system was determined from the experimental test data. This steady ratcheting strain was extrapolated to a design life of 350 hours. The extrapolated strain accumulation was checked against the ASME $1 \%$ limit. If the bars accumulate less than $1 \%$ strain in 350 hours they pass the design check, if they accumulate more than $1 \%$ strain they fail the check.

\subsection{Comparison to modified EPP strain limits procedure}

Table 5.1 also summarizes:

1. the results of an unmodified EPP strain limits calculation, using the unsoftened isochronous curves in the ASME code;

2. the results of the modified EPP strain limits procedure using the softening factors derived from the inelsatic model and summarized in Eq. 3.4;

3. the results of the modified EPP strain limits procedure using the experimental softening factors summarized in Table 4.1 and assuming that these factors for $600^{\circ} \mathrm{C}$ apply to all temperatures in the experimental test range and assuming the experimental reduction factor for the maximum number of cycles is the saturated factor.

For this particular set of experiments the existing, unmodified EPP strain limits procedure has a sufficient safety margin to cover any effect of cyclic softening. The modified procedures are also both conservative compared to the experimental results and both the numerically and experimentally calculated softening factors produce the same final results.

Two factors combine to make the unmodified EPP procedure conservative for these experiments. The first is that these experiments have relatively small amounts of cycling, relative to the hold time - on the order of 100-200 cycles (depending on delay time) in 350 hours. The second factor is the extrapolation procedure. The actual experimental test for each condition is only cycled until an apparent steady state is achieved. The final, 350 hour strain is extrapolated from these results. It is highly unlikely that the sample achieved a saturated softened state in less than 100 cycles and therefore most of the effect of softening will be omitted when using this procedure. 
temperature

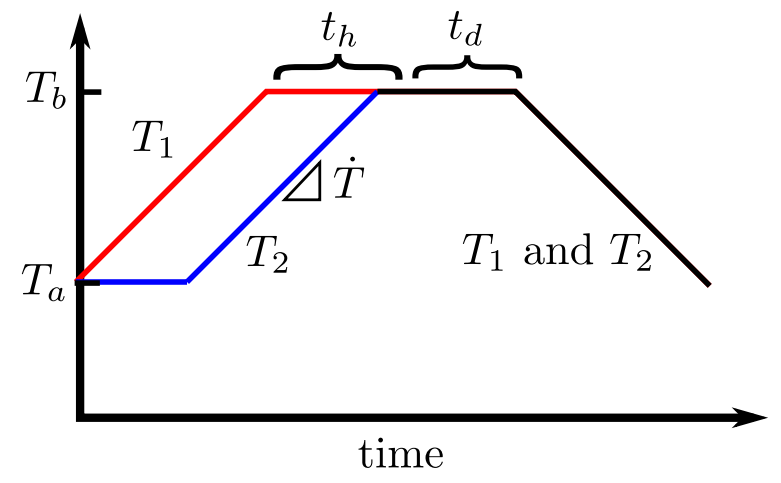

Figure 5.1: Two bar experimental thermal profile.

\begin{tabular}{cccccc}
\hline Load (lbs) & Delay $(\mathrm{min})$ & Exp. pass? & Unmod. EPP? & Mod. num. fac.? & Mod. exp. fac.? \\
\hline-100 & 10 & No & No & No & No \\
700 & 10 & Yes & Yes & Yes & Yes \\
900 & 10 & Yes & Yes & Yes & Yes \\
1150 & 10 & Yes & Yes & No & No \\
1400 & 10 & No & No & No & No \\
-400 & 10 & No & No & No & No \\
1700 & 8 & No & No & No & No \\
-400 & 6 & Yes & No & No & No \\
-700 & 5 & Yes & No & No & No \\
1700 & 3 & No & No & No & No \\
-1700 & 6.7 & No & No & No & No \\
\hline
\end{tabular}

Table 5.1: Comparison of experimental two bar test results to unmodified and modified EPP strain limits calculations. 
FY17 Status Report on the Initial EPP Finite Element Analysis of Grade 91 Steel

July 2017

Other experimental configurations and realistic component tests would result in cases where the unmodified EPP strain limits method is non-conservative, requiring the use of the modified procedure. Future work should identify simplified testing covering such cases and demonstrate the conservatism of the modified EPP strain limits procedure. 



\section{Conclusions and future work}

This report modifies the EPP strain limits design method to apply it to cyclic softening materials like Gr. 91. If the material softening saturates a further simplification of the modified method describes the softened material response with a set of temperature dependent, asymptotic softening factors. This simplified method could be implemented in an ASME code case to allow using the EPP strain limits method with Gr. 91 steel. The report demonstrates the conservatism of the modified method by comparing it to full inelastic simulations and a series of two bar experiments.

One issue raised in this report is the question of saturation in Gr. 91 cyclic response at high temperatures. At lower temperatures the experimental data shows saturating behavior - at least before the onset of damage. However, at higher temperatures softening does not seem to saturate. This could either be because the saturation time is very long at high temperatures and the material simply does not achieve a saturated state before beginning to develop damage or it could be some non-saturating, high temperature softening mechanism, distinct from the lower temperature behavior. Applying the simplified EPP strain limits method outlined here to Gr. 91 requires determining which one of these two behaviors is observed experimentally. This could be done with a series of interrupted fatigue tests, conducting microscopy to check for damage development and microstructural changes.

This report derives softening factors from inelastic models and from simplified experimental tests. The final proposed set of softening factors for Gr. 91 should be well-supported by the proposed test protocol - requiring tests spanning the full allowable Gr. 91 temperature range - or at least by a series of pre-softened creep tests designed to validate points on the softened isochronous curves. Furthermore, a similar experimental or model-based procedure must be devised to determine the softened yield stress of the material, as $S_{y}$ can determine the EPP pseudo-yield stress in some cases.

This report only considered the EPP strain limits code case and not the method for creep-fatigue damage evaluation. However, the methods are fundamentally similar and the creep-fatigue method relies on the same creep dissipation and work bounds also used by the strain limits methodology. Therefore, a similar modification to the creep-fatigue code case - using softened isochronous curves - should also be applied to the creep-fatigue approach. One open question is what effect cyclic softening might have on the fatigue and creep rupture life of the material. If it has a significant effect this might require a modification to the Gr. 91 creep-fatigue damage curves.

Modifications to the method are possible to reduce potential over-conservatism. As suggested above one possible modification would be to consider some intermediate, not-fullysaturated material state as the reference from which to derive softened isochronous curves.

Finally, the modified method presented here should be extensively tested against experimental results and simulations of realistic component geometries to thoroughly demonstrate its conservatism. The modified method could then serve as the basis of an ASME code case for applying the EPP methods to Grade 91 components. 



\section{Acknowledgments}

The research was sponsored by the U.S. Department of Energy (DOE), under Contract No. DE-AC02-06CH11357 with Argonne National Laboratory, managed and operated by UChicago Argonne LLC. Programmatic direction was provided by the Office of Advanced Reactor Technologies (ART) of the Office of Nuclear Energy (NE). The authors gratefully acknowledge the support provided by William Corwin of DOE-NE, ART Materials Technology Lead, and Robert Hill of Argonne National Laboratory, ART Co-National Technical Director.

The authors thank Yanli Wang at Oak Ridge National Laboratory for test results and consultation on the modified EPP procedure. They also thank Bob Jetter of R.I. Jetter Consulting and Peter Carter of Stress Engineering Services, Inc., for discussions on the EPP and ASME code design methodologies. Finally, they thank David Parks of MIT for his comments and corrections to the proofs in Section 2. 



\section{Bibliography}

[1] F. Abe, S. Nakazawa, H. Araki, and T. Noda. The role of microstructural instability on creep behavior of a martensitic 9Cr-2W steel. Metallurgical Transactions A, 23(2): 469-477, 1992.

[2] R. A. Ainsworth. Bounding solutions for creeping structures subjected to load variations above the shakedown limit. International Journal of Solids and Structures, 15(12):981986, 1979.

[3] R. A. Ainsworth. Appplication of bounds for creeping structures subjected to load variations above the shakedown limit. International Journal of Solids and Structures, 13:981-993, 1979.

[4] R. A. Ainsworth. A note on bounding solutions for creeping structures subjected to load variations above the shakedown limit. International Journal of Solids and Structures, 15(12):981-986, 1979.

[5] American Society of Mechanical Engineers. Case N-861: Satisfaction of Strain Limits for Division 5 Class A Components at Elevated Temperature Service Using Elastic-Perfectly Plastic Analysis. In ASME Boiler and Pressure Vessel Code, Nuclear Component Code Cases. 2015.

[6] American Society of Mechanical Engineers. Case N-862: Calculation of Creep-Fatigue for Division 5 Class A Components at Elevated Temperature Service Using ElasticPerfectly Plastic Analysis. In ASME Boiler and Pressure Vessel Code, Nuclear Component Code Cases. 2015.

[7] American Society of Mechanical Engineers. Section III, Division 5: High Temperature Reactors. In ASME Boiler and Pressure Vessel Code. 2015.

[8] J. T. Boyle. The behaviour of creeping structures at large times. Journal de Mecanique, 15:195, 1976.

[9] P. Carter. Bounding theorems for creep-plasticity. International Journal of Solids and Structures, 21(6):527-543, 1985.

[10] Peter Carter. Analysis of cyclic creep and rupture. Part 1: Bounding theorems and cyclic reference stresses. International Journal of Pressure Vessels and Piping, 82(1): 15-26, 2005.

[11] Peter Carter. Analysis of cyclic creep and rupture. Part 2: Calculation of cyclic reference stresses and ratcheting interaction diagrams. International Journal of Pressure Vessels and Piping, 82(1):27-33, 2005.

[12] Peter Carter, T.-L. Sham, and R. I. Jetter. Simplified analysis methods for primary load designs at elevated temperatures. In Proceedings of the ASME 2011 Pressure Vessel \& Piping Division Conference, 2011. 
[13] Peter Carter, Robert I. Jetter, and T.-L. Sham. Application of elastic-perfectly plastic cyclic analysis to assessment of creep strain. In Proceedings of the ASME 2012 Pressure Vessels \& Piping Division Conference, 2012.

[14] Peter Carter, R. I. Jetter, and T.-L. Sham. Verification of elastic-perfectly plastic methods for evaluation of strain limits - analytical comparisons. In Proceedings of the ASME 2014 Pressure Vessels \& Piping Conference, pages 1-8, 2014.

[15] E Cerri, E Evangelista, S Spigarelli, and P Bianchi. Evolution of microstructure in a modified 9Cr-1Mo steel during short term creep. Materials Science and Engineering: A, 245(2):285-292, 1998.

[16] C. O. Frederick and P. J. Armstrong. Convergent internal stresses and steady cyclic states of stress. The Journal of Strain Analysis for Engineering Design, 1(2):154-159, 1966.

[17] S Kim and J R Weertman. Investigation of Microstructural Changes in a Ferritic Steel Caused by High Temperature Fatigue. Metallurgical Transactions, 19A(April):999-1007, 1988.

[18] K. Kimura, H. Kushima, and K. Sawada. Long-term creep deformation property of modified 9Cr-1Mo steel. Materials Science and Engineering A, 510-511(C):58-63, 2009.

[19] F. A. Leckie and J. B. Martin. Deformation bounds for bodies in a state of creep. Journal of Applied Mechanics, 34(2):411-417, 1967.

[20] Z. Mroz. On the theory of steady plastic cycles in structures. In First SMiRT Conference, page L 5/6, 1971.

[21] A. R. S. Ponter. Deformation, displacement, and work bounds for structures in a state of creep and subject to variable loading. Journal of Applied Mechanics, 39:953, 1972.

[22] A. R. S. Ponter. General bounding theorems for the quasi-static deformation of a body of inelastic materia, with applications to metallic creep. Journal of Applied Mechanics, 41:947, 1974.

[23] A. R S Ponter. General displacement and work bounds for dynamically loaded bodies. Journal of the Mechanics and Physics of Solids, 23(2):151-163, 1975.

[24] Vani Shankar, M. Valsan, K. Bhanu Sankara Rao, R. Kannan, S. L. Mannan, and S. D. Pathak. Low cycle fatigue behavior and microstructural evolution of modified 9Cr-1Mo ferritic steel. Materials Science and Engineering A, 437(2):413-422, 2006.

[25] V. K. Sikka, C. T. Ward, and K. C. Thomas. Modified 9Cr-1Mo steel-an improved alloy for steam generator application. In Proceedings of the ASM International Conference on Production, Fabrication Properties, and Applications of Ferritic Steels for High Temperature Applications, 1982. 
[26] J. R. Stefano, V. K. Sikka, J. J. Blass, C. R. Brinkman, J. M. Corum, J. A. Horsk, R. L. Huddleston, J. F. King, R. W. McClung, and W. K. Sartory. Summary of Modified 9Cr-1Mo Development Program: 1975-1985. Technical Report ORNL-6303, OSTI ID 712852, Oak Ridge National Laboratory, 1986.

[27] R. W. Swindeman, M. L. Santella, P. J. Maziasz, B. W. Roberts, and K. Coleman. Issues in replacing Cr-Mo steels and stainless steels with $9 \mathrm{Cr}-1 \mathrm{Mo}-\mathrm{V}$ steel. International Journal of Pressure Vessels and Piping, 81(6):507-512, 2004.

[28] Yanli Wang, Robert I Jetter, and T.-L. Sham. Progress report on the development of test procedure for the two-bar thermal ratcheting experiment for Alloy 617. Technical Report ORNL/TM-2013/318, 2013.

[29] Yanli Wang, Robert I Jetter, Seth T Baird, Chao Pu, and T.-L. Sham. Report on FY15 Two-Bar Thermal Ratcheting Test Results. Technical Report ORNL/TM-2015/284 OSTI ID 1223078, 2015.

[30] M. Yaguchi and Y. Takahashi. Unified Inelastic Constitutive Model for Modified 9Cr1Mo Steel Incorporating Dynamic Strain Aging Effect. JSME International Journal, Series A, 42(1):1-10, 1999.

[31] Masatsugu Yaguchi and Yukio Takahashi. A viscoplastic constitutive model incorporating dynamic strain aging effect during cyclic deformation conditions. International Journal of Plasticity, 16(3-4):241-262, 2000. 

FY17 Status Report on the Initial EPP Finite Element Analysis of Grade 91 Steel July 2017

\section{Distribution List}

$\begin{array}{lll}\text { Name } & \text { Affiliation } & \text { Email } \\ \text { Corwin, W. } & \text { DOE } & \text { william.corwin@nuclear.energy.gov } \\ \text { Sowinski, T. E. } & \text { DOE } & \text { thomas.sowinski@nuclear.energy.gov } \\ \text { Grandy, C. } & \text { ANL } & \text { cgrandy@anl.gov } \\ \text { Hill, R. N. } & \text { ANL } & \text { bobhill@anl.gov } \\ \text { Li, M. } & \text { ANL } & \text { mli@anl.gov } \\ \text { Messner, M. C. } & \text { ANL } & \text { messner@anl.gov } \\ \text { Natesan, K. } & \text { ANL } & \text { natesan@anl.gov } \\ \text { Sham, T.-L. } & \text { ANL } & \text { ssham@anl.gov } \\ \text { Wang, Y. } & \text { ORNL } & \text { wangy2@ornl.gov } \\ \text { Wright, R. N. } & \text { INL } & \text { richard.wright@inl.gov }\end{array}$






\section{Argonne}

\section{Nuclear Engineering Division}

Argonne National Laboratory

9700 South Cass Avenue, Bldg. 208

Argonne, IL 60439

www.anl.gov 